\title{
THE EVOLUTIONARY GENETICS OF CANALIZATION
}

\author{
Thomas Flatt
}

Division of Biology and Medicine, Department of Ecology and Evolutionary Biology, Brown University,

\section{Keywords}

canalization, constraint, epistasis, genetic architecture, genetic assimilation, genetic variance, genotype by environment interaction, genotype-

phenotype, map, phenotypic plasticity, phenotypic variance, selection

\begin{abstract}
Evolutionary genetics has recently made enormous progress in understanding how genetic variation maps into phenotypic variation. However, why some traits are phenotypically invariant despite appar-ent genetic and environmental changes has remained a major puzzle. In the 1940s, Conrad Hal Waddington coined the concept and term "canalization" to describe the robustness of phenotypes to perturbation; a similar concept was proposed by Waddington's contemporary Ivan Ivanovich Schmal-hausen. This paper reviews what has been learned about canalization since Waddington. Canalization implies that a genotype's phenotype remains relatively invariant when individuals of a particular genotype are exposed to different environments (environmental canalization) or when individuals of the same single- or multilocus genotype differ in their genetic background (genetic canalization). Con-sequently, genetic canalization can be viewed as a particular kind of epistasis, and environmental canalization and phenotypic plasticity are two aspects of the same phenomenon. Canalization results in the accumulation of phenotypically cryptic genetic variation, which can be released after a "decan-alizing" event. Thus, canalized genotypes maintain a cryptic potential for expressing particular phe-notypes, which are only uncovered under particular decanalizing environmental or genetic conditions. Selection may then act on this newly released genetic variation. The accumulation of cryptic genetic variation by canalization may therefore increase evolvability at the population level by leading to phenotypic diversification under decanalizing conditions. On the other hand, under canalizing con-ditions, a major part of the segregating genetic variation may remain phenotypically cryptic; canalization may therefore, at least temporarily, constrain phenotypic evolution. Mechanistically, canalization can be understood in terms of transmission patterns, such as epistasis, pleiotropy, and genotype by environment interactions, and in terms of genetic redundancy, modularity, and emergent properties of gene networks and biochemical pathways. While different forms of selection can favor canalization, the requirements for its evolution are typically rather restrictive. Although there are several methods to detect canalization, there are still serious problems with unambiguously demonstrating canalization, particularly its adaptive value.
\end{abstract}


$\mathrm{T}$ HE STUDY of phenotypic variation is a central theme in evolutionary biology. Natural selection results from variation in fitness among individuals; the response to selection depends on the heritable determinants of the phenotype. Phenotypes are the product of developmental processes that depend both on the genotype and environment and their interaction. It is thus important to understand how genetic variation maps to phenotypic variation, and how this genotypephenotype map is influenced by genetic and environmental change. Although evolutionary biology is to a major degree concerned with the study of variation, we have only a limited understanding of the absence of variation. Why are some traits phenotypically invariant despite apparent genetic and environmental changes? Why are some traits less phenotypically variable in some taxa, but not in others?

One of the most intriguing observations in evolutionary genetics is that wild-type populations often harbor vast amounts of hidden genetic variation, this variation being phenotypically expressed only in particular environments or genetic backgrounds (e.g., Gibson et al. 1999; Gibson and Dworkin 2004). Thus, there seems to be a strong robustness of some phenotypes against genetic and nongenetic change or perturbation. More generally, the amount and quality of phenotypic variation can differ dramatically within and among populations. Some traits are highly invariant within species while simultaneously being highly variable among closely related species; other characters seem to be highly conserved among species or clades. Why is this the case? Similarly, the same trait may be more variable in some taxa than in others. Does robustness occur in all taxa and for all traits to the same extent? Some of the differences in the amount and quality of pheno- typic variation may well be explained by classical scenarios, such as selection or drift. Yet, it remains a fascinating possibility that organisms may have evolved specific mechanisms that make them insensitive to genetic and environmental change, thereby decreasing their capacity for evolutionary change. Alternatively, the molecular details of the functional architecture of complex phenotypic traits may lead to robustness as a nonadaptive byproduct, an emergent property. This commonly observed robustness of phenotypes was named "canalization" by Waddington in the 1940s to describe the mechanisms that cause the phenotype to be insensitive against genetic and nongenetic perturbations and change (Waddington 1942; see also Schmalhausen 1949).

Canalization is highly relevant for evolutionary biology. For example, it implies that phenotypes may be stable around their fitness optimum despite genetic and environmental change (e.g., Rendel 1967). By keeping phenotypic variation low, canalization may constrain phenotypic evolution (e.g., Charlesworth et al. 1982; Maynard Smith et al. 1985) and provide a microevolutionary mechanism for character stasis (e.g., Stearns 1994). Canalization also allows genetic variation that is phenotypically not expressed to accumulate. This cryptic variation can lead to the appearance of new phenotypes when development is "decanalized," for instance by environmental stress, thereby allowing evolutionary change (e.g., Rutherford and Lindquist 1998).

Despite the long history of the canalization concept (Waddington 1942; Schmalhausen 1949; also see Hall 1992; Gilbert 2000; Slack 2002), the evidence for canalization is limited (e.g., Scharloo 1991; Gibson and G P Wagner 2000; De Visser 2003). The evolutionary role of canalization has remained puzzling: the 
concept is difficult to define, and its predictions are often beyond the reach of experiments. Similarly, the molecular mechanisms governing canalization are poorly understood. Thus, not surprisingly, following the classical work by Waddington, Schmalhausen, and others, empirical and theoretical research on canalization declined, presumably due to the lack of suitable theoretical and genetic methods to tackle the problem of phenotypic robustness. However, recent advances in molecular developmental genetics and theoretical biology have set the stage for a comeback of the canalization concept among theoretical biologists (cf. Gibson and G P Wagner 2000), evolutionary geneticists (cf. De Visser et al. 2003), and developmental biologists (cf. Gerhart and Kirschner 1997; Hartman et al. 2001). Thus, recent theoretical work (e.g., G P Wagner et al. 1997; Rice 1998; Kawecki 2000; Siegal and Bergman 2002; Bergman and Siegal 2003; Hermisson et al. 2003; Hermisson and G P Wagner 2004; Proulx and Phillips 2005), evolutionary experiments (e.g., Stearns and Kawecki 1994; Stearns et al. 1995; Elena and Lenski 2001), and molecular studies (e.g., Gibson and Hogness 1996; Rutherford and Lindquist 1998; True and Lindquist 2000; A Wagner 2000b; Queitsch et al. 2002; Gu et al. 2003; Sollars et al. 2003; True et al. 2004) have renewed interest in canalization.

This paper provides a comprehensive review of what has been learned about the evolutionary genetics of canalization since Waddington (for recent, shorter reviews, see Gibson and G P Wagner 2000; Meiklejohn and Hartl 2002; De Visser et al. 2003; Gibson and Dworkin 2004; also see the book edited by Hall and Olson 2003). I ask six questions: (1) How can canalization be defined? (2) What is the relationship between canalization, epistasis, and genotype by environment interactions? (3) At the proximate level, which molecular mechanisms may lead to canalization? (4) At the ultimate level, how does canalization originate? Is it a sideproduct or an emergent property of the genotypephenotype map, or is it shaped by natural selection? (5) What are the consequences of canalization for evolutionary processes? (6) How can canalization be measured?
One of the most fundamental problems of evolutionary biology is to better understand the pathways that connect genotypes with phenotypes, the genotype-phenotype map (e.g., Lewontin 1974a; Wright 1977; Houle 1991, 2001; Schlichting and Pigliucci 1998). Canalization is but one aspect of the genotype-phenotype map; the general problem is to understand the functional architecture of complex phenotypes that depend upon manifold interactions among underlying genes and the environment. A better understanding of canalization will contribute to a clearer conceptual picture of how genes, development, and the environment interact to produce phenotypes.

\section{The Phenomenon of Canalization}

\section{THE STABILITY OF THE WILD-TYPE}

Waddington based the concept of canalization on the observation that genotypes differ in their phenotypic reactions to genetic and environmental change, and that wildtype phenotypes are phenotypically much less variable than mutants or environmentallyinduced phenotypes (Waddington 1942; Gibson et al. 1999). In a similar vein, Schmalhausen (1949) argued that the "stability of the morphogenetic system is destroyed (rendered labile) due either to variation in environmental factors or to mutation" (Schmalhausen 1949/1986:79). Waddington (1957) suggested that the reason for the difference in variation between wild-type as opposed to mutant or environmentally-induced phenotypes is that the wild-type has been exposed to many generations of stabilizing selection, whereas the mutant has not (see also Schmalhausen 1949). Several experiments seem to support Waddington's view by showing that stabilizing selection can reduce the variability of environmentally-induced or mutant phenotypes, and that phenotypic change becomes progressively more difficult when the trait approaches the wild-type pattern (e.g., Maynard Smith and Sondhi 1960; Waddington 1960). This has been taken as evidence for the adaptive canalization of the wild-type (cf. Scharloo 1991). However, in most experiments it is not clear whether stabilizing selection just decreased genetic variation or 
whether it selected for canalizing mechanisms itself. Furthermore, the wild-type concept is an ambiguous abstraction since natural wild-type populations are often highly genetically and phenotypically variable. See Scharloo (1991) for a comprehensive review of Waddington's original view.

\section{DEFINITION OF CANALIZATION}

Canalization is the reduced sensitivity of a phenotype to changes or perturbations in the underlying genetic and nongenetic factors that determine its expression (see also Meiklejohn and Hartl 2002; De Visser et al. 2003). Canalization is a relative term, and can thus only be defined as a matter of comparison. Thus, a phenotype $\mathrm{P}$ is more canalized than another phenotype $\mathrm{P} *$ if $\mathrm{P}$ remains relatively invariant when the single- or multilocus genotype $G$, which determines $P$, is exposed to different environments (environmental canalization) or located in different genetic backgrounds (genetic canalization): $\mathrm{P}$ is "resilient," "robust," or "insensitive" to genetic and/or environmental changes or perturbations. Canalization can therefore be recognized by observing that most genetic or environmental changes leave the phenotypic expression of $G$, and thus the phenotype $P$, invariant; the expression of $G$ is changed such that specific phenotypic changes $\left(\mathrm{P} \rightarrow \mathrm{P}^{*}\right)$ are induced only in some genetic backgrounds or environments (or combinations of genetic backgrounds and environments). Consequently, a canalizing allele or genotype G reduces the phenotypic variation of a trait across a range of genetic backgrounds and environments relative to a noncanalizing allele or genotype $G^{*}$, and a canalized trait $P$ exhibits a restricted range of phenotypic variation across genetic backgrounds and environments as compared to a noncanalized trait $\mathrm{P}^{*}$ (Meiklejohn and Hartl 2002).

The proximate (molecular) mechanisms causing canalization, as well as the nature of the perturbations, can be manifold. For example, the canalizing mechanisms can potentially be located at any level of the biological hierarchy, from gene expression, RNA stability, protein structure and folding, intermediate metabolism and physiology to mor- phology, behavior, and life-history traits. For instance, canalization may be observed at the level of morphology, but not at the level of gene expression. The buffering would then occur at some intermediate level between gene expression and morphology. Furthermore, to maintain a particular trait (e.g., body temperature in homeotherms) despite (e.g., thermal) perturbations, an organism may vary other traits (such as basal metabolic rate and dilation of blood vessels). Stability (homeostasis) at one level may depend upon lability or sensitivity at another level. Thus, it seems that there exists an intrinsic paradox of canalization: buffering at one level (the phenotype) may be coupled to higher variation at another level (e.g., gene expression). Variation in such buffering mechanisms can be heritable or nonheritable.

Homology, when two or more structures are alike because of shared ancestry, may be another interesting aspect of the biological hierarchy related to canalization. Homology can occur between entities at different levels of the biological hierarchy; for instance some organisms may show homologies at the genetic level, but not necessarily at the morphological level (Laubichler 2000). Consequently, individuals from different species are often composed of the same kind of (structurally identical) building blocks (e.g., genes, organs, and traits). Such homologous structures may be a manifestation of canalization; canalization may potentially explain the stability of homologues, since canalization may limit or constrain variation in these structures (e.g., G P Wagner 1996; G P Wagner and Altenberg 1996; Laubichler 2000). A similar concept to homology is homoplasy, when two or more structures are alike but not due to common ancestry. It is an interesting, yet open empirical question whether and how canalization affects homology and homoplasy and whether, for example, homoplasy is more commonly observed in taxa with less canalized traits.

Various authors have used different terminologies when referring to canalization or aspects of it (see Debat and David 2001; Meiklejohn and Hartl 2002). Autonomous development or autoregulation (Schmalhausen 1938, 1949), homeostasis, homeorhesis or buf- 
fering (Bernard 1865; Cannon 1932; Lerner 1954; Lewontin 1956; Zakharov 1992; Kauffman 1993; Hallgrímsson et al. 2002), developmental stability (Thoday 1955; Palmer and Strobeck 1986, 1992), epigenetic stability (A Wagner 1996), and robustness (e.g., Savageau 1971; Kauffman 1993; Little et al. 1999; De Visser et al. 2003) are essentially synonymous with canalization. Sometimes canalization is taken to mean adaptive, evolved robustness against heritable and nonheritable perturbations, whereas terms such as buffering refer to any kind of mechanism, adaptive or not, that will cause the phenotype to be resilient against perturbations (cf. DeVisser et al. 2003). See Hall and Olson (2003) for an excellent recent discussion of evolutionary developmental biology in general, including various issues bearing on canalization discussed in this review. Here I use the term canalization to mean any mechanism, structure, or process, adaptive or not, that will reduce a phenotype's sensitivity to perturbations.

\section{AN EXAMPLE OF CANALIZATION}

When the function of the Hsp90 protein, a chaperone and heat shock protein, encoded by the $h s p 83$ locus in Drosophila melanogaster is impaired by mutation or by the specific inhibitor geldanamycin, phenotypic variation increases both in laboratory and wild strains (Rutherford and Lindquist 1998). The degree of increase and the nature of the phenotypic variation depend on the genetic background and environment (e.g., temperature). A wide range of phenotypic effects is observed, including defects in bristles, eyes, halteres, legs, wings, the thorax, and abdomen. The authors showed that at least some of these phenotypes are produced by alleles that are not phenotypically expressed in the presence of the functional Hsp90 protein. This genetic variation is heritable, and the pattern of heritability is unlikely to be due to de novo mutation but to genetic variance in polygenic traits. Thus, impairment of Hsp90 uncovers previously silent genetic variation, which leads to an increase of phenotypic variation (decanalization). When decanalized lines, showing a high penetrance of a par- ticular trait, are outcrossed with normal laboratory strains, the trait is expressed only at very low levels, implying a return to the canalized state. Thus, the functional $h s p 83$ locus is a gene with canalizing effects, masking the effects of hidden genetic variation. Queitsch et al. (2002) confirmed the results of Rutherford and Lindquist (1998) by showing that reducing the function of Hsp90 in various Arabidopsis genotypes increases genetic variation in morphological traits.

But does Hsp90 also buffer against environmental, nongenetic perturbations such as developmental noise? As work by Milton et al. (2003) demonstrates, this does not seem to be the case (but see Queitsch et al. 2002): in D. melanogaster, Hsp90 does not buffer against nongenetic perturbations as measured by fluctuating asymmetry in bristle traits. The Hsp90 system is undoubtedly the best current example of a molecular canalizing mechanism; it nicely illustrates how environmental cues (e.g., geldanamycin, temperature) or mutation can lead to the release of hidden genetic variation. Intriguingly, Sollars et al. (2003) have shown that Hsp90 can act through epigenetic mechanisms, whereby a reduced activity of Hsp90 causes a heritable change in the chromatin state (see True et al. 2004 for another example of epigenetic canalization).

The importance of heat shock proteins for canalization has also been confirmed by Fares et al. (2002): the authors show that overexpression of the heat shock protein GroEL induces the recovery of fitness of Escherichia coli strains that have accumulated deleterious mutations. Yet, recent modeling work by Hermisson and G P Wagner (2004) casts some doubt on whether Hsp90 can really be seen as an example of canalization. Although canalization is an attractive explanation for the accumulation of cryptic genetic variation, theory clearly shows that canalization is not necessarily required to explain the buildup of cryptic variation. Accumulation of mutations at conditionally neutral loci for a sufficiently long time can lead to the accumulation of cryptic variation even in the absence of canalization (Hermisson and G P Wagner 2004). See Rutherford (2003) for a comprehensive review of the role of protein chaperones such 
as the Hsp family for canalization and buffering; see Scharloo (1991) for a review of some of the classic-but more ambiguousexamples of canalization.

\section{POTENTIAL VARIATION}

Canalization has been defined as a state of reduced variability, that is, highly canalized genotypes show a reduced potential to vary in response to genetic or environmental change as compared to some less canalized mutants or environmentally induced phenotypes (G P Wagner and Altenberg 1996; Gibson and van Helden 1997; G P Wagner et al. 1997; Gibson and G P Wagner 2000). Thus, the concept of "variability" (not to be mistaken by the term "variation," which refers to the actual level of change) is a way of expressing the observation that highly canalized genotypes are much more insensitive to mutations or environmental changes than most other, less canalized genotypes (G P Wagner and Altenberg 1996; G P Wagner et al. 1997; Gibson and G P Wagner 2000). Thus, in comparison, genotypes with high variability (reduced state of canalization) change their phenotype much more readily than genotypes with low variability (increased state of canalization) when faced by the same mutational or environmental change. Variability is itself under genetic control; some genotypes are more canalized than others. For example, genetic polymorphisms at the Ultrabithorax (Ubx) locus in Drosophila melanogaster, a gene required for the establishment of thoracic segment identity, have been shown to be responsible for the differential insensitivity among $U b x$ genotypes against developmental perturbations by ether vapor (Gibson and Hogness 1996).

Canalization uncouples genetic variation from phenotypic variation among the canalized genotypes in the population. This is because, in contrast to weakly or noncanalized genotypes, highly canalized genotypes can accumulate mutations or maintain existing alleles that are not phenotypically expressed. Thus, part of the genetic variation in the population can be maintained in a hidden form. For example, wild-type genotypes often harbor substantial amounts of cryptic or hidden genetic variation (Gibson et al.
1999; Gibson and Dworkin 2004), which are only expressed when the genetic background changes-a common observation also made by developmental geneticists working on specific mutations in different genetic backgrounds. Similarly, in a mutation accumulation experiment, Davies et al. (1999) exposed a wild-type strain of Caenorhabditis elegans to a mutagen and, from this strain, bred sixty independent lines toward homozygosity by selfing. In fitness assays of these lines, the authors found that the frequency of deleterious mutations is about $96 \%$, and these mutations have fitness effects of less than $0.07 \%$. Yet, whether these fitness effects are reduced due to canalization, that is, they are magnified in other environments or genetic backgrounds, remains to be determined. In haploid mutant strains of yeast (Saccharomyces cerevisiae), Thatcher et al. (1998) found that a major proportion of null mutations has almost no effect on fitness because the function of a given knocked-out gene can be compensated by other genes. Thus, there is genetic variation due to null mutations with potentially deleterious effects on fitness, but this variation is cryptic and has no phenotypic consequences for fitness. For a recent review on cryptic genetic variation see Gibson and Dworkin (2004).

The cryptic pool of genetic variation accumulated under canalization can be phenotypically expressed again if genetic or environmental change uncovers the silent genetic variation (decanalization; e.g., Scharloo 1991; Gibson and Hogness 1996; Rutherford and Lindquist 1998; Gibson et al. 1999; Gibson and Dworkin 2004; Hermisson and G P Wagner 2004), thereby increasing phenotypic variation in the population. Decanalizing conditions can be due to environmental perturbations that change environment-dependent gene expression or allele substitutions that render canalizing mechanisms nonfunctional. Whether a state of decanalization will be reached is likely to depend on the degree of canalization of a genotype or population as well as on the frequency and intensity of the decanalizing conditions. Interestingly, recent theory shows that mutation accumulation may eventually lead to a state of decanalization and indicates that a state of 
high variability may be a generic property of mutant phenotypes. This is because under most forms of epistasis, mutation accumulation is expected to lead to an accelerating increase of additive genetic variance (Hansen and G P Wagner 2001). Consider the effect of a single major mutation on the mutational variance of a particular trait in a given genetic background. It has been found that the stronger the epistasis (i.e., the stronger the effect of one locus on a substitution at another locus), the stronger is the increase in additive genetic variance under mutation accumulation (Hansen and G P Wagner 2001). Thus, mutations are decanalizing; the more mutations that have already accumulated and the stronger the epistasis, the more decanalizing the mutations are (Hansen and G P Wagner 2001). Thus, the results suggest that a state of increased variability of mutant phenotypes may be a generic property of epistatic systems (Hansen and G P Wagner 2001). As discussed by these authors, this finding does not falsify the concept of canalization, but eliminates increased variability of mutant phenotypes as indirect evidence for the wild-type being canalized to reduce the effects of genetic and environmental changes, as had been suggested by Waddington (1942). Recent work supports this notion by showing that the release of genetic variation caused by mutation or environmental perturbation is a generic property of systems exhibiting epistasis or genotype by environment interactions (Hermisson and G P Wagner 2004).

\section{Genetic And Environmental Canalization}

A useful way to illustrate canalization is to consider a phenotypic landscape (Rice 1998, 2000, 2002; Wolf et al. 2001; Nijhout 2002). A phenotypic landscape is a surface that defines the phenotype $\phi$ as a smooth function of underlying factors, for example, genetic and environmental factors (Rice 1998, 2000, 2002). Interactions among these factors represent the process of development and determine the shape of the phenotypic landscape. The height of the landscape is a measure of some phenotypic trait, and the different contour lines of the landscape represent differ- ent phenotypic values: along a given contour line, the underlying factors produce the same phenotypic value. The spacing between contours of equal phenotype indicates the slope of the surface. At a given point, the slope along the contour indicates the degree to which a given amount of variation in the underlying factors translates into phenotypic variation. The slope is therefore a measure of the degree of canalization. If the underlying factors contribute additively to the phenotype, then the landscape is a plane with no curvature, the degree of canalization being everywhere the same. If the factors contribute nonadditively (e.g., epistasis, genotype by environment interactions), then the landscape is curved, and there are different degrees of canalization (as represented by the unequal spacing of contour lines). If the surface curves, then the slope is likely to be lower at some points along a contour than at others. The points of minimal slope along a given contour line are the points of maximum canalization for a given phenotype. In contrast, points of equal canalization along a contour line have the same partial derivative of the phenotype $\phi$ with respect to a given change in an underlying factor.

This geometrical view makes two important assumptions. First, the phenotypic landscape is a function of several underlying factors that determine the expression of the phenotype. Thus, based on the source of perturbation against which the phenotype is buffered, one can distinguish, for example, between genetic and environmental canalization (e.g., Waddington 1942, 1957; Stearns and Kawecki 1994; G P Wagner et al. 1997). Second, nonadditivity of the factors shaping the phenotypic landscape implies different degrees of canalization. Thus, canalization is best described in terms of nonadditive interactions, such as epistasis and genotype by environment interactions.

\section{GENETIC CANALIZATION AND EPISTASIS}

Epistasis is the influence of one locus on the expression of genetic variance at another locus (Moreno 1994; Whitlock et al. 1995; Fenster et al. 1997; Wolf et al. 2000; Wade et al. 2001). A wealth of genetic observations 
indicates that epistatic effects are extremely common: genes may have specific effects on particular phenotypes, but these effects often strongly depend on other interacting genes (Wade 2002). For example, wild-type genotypes often have large amounts of cryptic genetic variation, which is only phenotypically expressed in some but not in other genetic backgrounds (Gibson et al. 1999). Consequently, specific genetic effects on the phenotype may be absent, weaker, or stronger, depending on the genetic background. Genetic canalization means that allele substitutions with a potential for phenotypic change in some genetic backgrounds are not expressed in other genetic backgrounds. Thus, genetic canalization is an epistatic phenomenon (buffering or canalizing epistasis) (Nijhout and Paulsen 1997; G P Wagner et al. 1997; Rice 2000; Hansen and G P Wagner 2001; Wade et al. 2001; De Visser et al. 2003; Burch and Chao 2004); yet, as I will discuss later, pleiotropy, the effect of a single gene or allele on two or more traits, is another potentially important property of the genotype relevant to canalization.

By decreasing the effects of other loci, these epistatic effects allow an increase of genetic variation that is not phenotypically expressed. Consequently, genetic canalization reduces the mutational variance $V_{M}$, the phenotypic variance caused by the input of recurrent mutations (G $P$ Wagner et al. 1997). Thus, the inverse of the mutational variance can be taken as a measure of genetic canalization or mutational robustness. Since epistasis means that different genotypes react differently to allele substitutions, that is, more or less sensitively, its presence implies different degrees of genetic canalization. In the broadest sense, genetic canalization refers to the robustness against both heritable genetic as well as epigenetic perturbations (Sollars et al. 2003).

It is also noteworthy that genetic canalization can lead to genetic neutrality (A Wagner 1996; Nijhout and Paulsen 1997; Fontana and Schuster 1998; Bornholdt and Sneppen 2000; De Visser et al. 2003): canalization allows the accumulation of phenotypically silent or neutral mutations, which can alter the genetic basis of a trait. This may set the stage for the accumulation of phenotypically relevant mutations (see Fontana and Schuster 1998; De Visser et al. 2003). Interestingly, as shown by Dworkin et al. (2003), the gene for the epidermal growth factor receptor (Egfr) harbors synonymous substitutions that supply cryptic genetic variation for photoreceptor determination. Remarkably, this variation, not observed in most environmental conditions or genetic backgrounds, shows the imprint of purifying selection (Dworkin et al. 2003). Thus, the cryptic variation supplied by synonymous substitutions can, depending on the context, lead to the expression of phenotypic variation visible to selection (Dworkin et al. 2003). A more detailed review of the concept of genetic canalization or robustness can be found in De Visser et al. (2003).

\section{ENVIRONMENTAL CANALIZATION AND PHENOTYPIC PLASTICITY}

Phenotypic plasticity is the sensitivity of the phenotype produced by a single genotype to variation in the environment (e.g., Stearns 1989a; Roff 1997). In contrast, environmental canalization is the insensitivity of a phenotype to variation in the environment; in the broad sense, environmental canalization refers to any kind of robustness against nonheritable perturbations (Waddington 1942, 1957; Roff 1997; De Visser et al. 2003). Thus, environmental canalization and phenotypic plasticity describe different aspects of the same phenomenon: the dependency of the phenotype on the environment (e.g., Stearns 1982; Palmer 1994; Roff 1994, 1997; Ancel and Fontana 2000; Rice 2000; Rutherford 2000; De Visser et al. 2003; Proulx and Phillips 2004; see Debat and David 2001 for a discussion). This can also be seen by noting that phenotypic plasticity of one trait often causes environmental canalization for another trait.

The environmental perturbations to which a phenotype can be insensitive may either be external environmental factors (e.g., temperature) or internal environmental factors (e.g., developmental noise); both kinds of perturbation are nonheritable. In quantitative genetics, the phenotypic variation caused by variation in both external and internal, nonheritable environmental factors is sub- 
sumed in the environmental variance component, $V_{E}$. Thus, the simplest measure of environmental canalization is the inverse of $\mathrm{V}_{\mathrm{E}}$.

Environmental canalization, often called development stability, has been defined by some authors as phenotypic insensitivity to microenvironmental perturbations (G P Wagner et al. 1997), whereas the concept of phenotypic plasticity has usually been applied to phenotypic sensitivity to macroenvironments (Scheiner 1993). However, the concepts of environmental canalization and phenotypic plasticity can be applied to variation in both micro- and macroenvironments (Via 1994; Schlichting and Pigliucci 1998; Debat and David 2001).

\section{MACROENVIRONMENTAL CANALIZATION}

Macroenvironments are shared by many individuals (e.g., climate conditions, habitat differences, food levels). Macroenvironmental phenotypic plasticity is phenotypic sensitivity to macroenvironments. It is classically described with the concept of a reaction norm, the set of all phenotypes produced by a genotype across a set of environments (Woltereck 1909; Schmalhausen 1949; Falk 2001). By analogy, macroenvironmental canalization is phenotypic insensitivity to macroenvironments. A genotype whose reaction norm is less steep than that of another genotype is macroenvironmentally more canalized, that is, less plastic. Similarly, a reaction norm can exhibit a zone of canalization, a region of the reaction norm that is relatively flat where changes in the environment result in relatively little phenotypic change. Variation in the slopes of reaction norms implies different degrees of macroenvironmental canalization. At present, we do not know much about the patterns of macroenvironmental canalization, for example, how frequently flat reaction norms or zones of canalization occur. If we knew more about this, we could, for instance, address questions about which environmental ranges favor plasticity and which favor environmental canalization. This would tell us a lot about selection for optimal reaction norms (Stearns and Koella 1986).

\section{MICROENVIRONMENTAL CANALIZATION}

Microenvironments are specific for a given individual since there are unpredictable errors in the development of an individual (developmental noise). Those errors may represent responses to random environmental fluctuations immediately external to that individual or nongenetic internal changes in processes sensitive to chance fluctuations in molecule numbers (McAdams and Arkin 1997; Goss and Peccoud 1998). We do not yet know whether the patterns that have been characterized as microenvironmental are in fact due primarily to variation in the external environment or to nongenetic variation within the organism, whether the perturbations are external or internal (see Debat and David 2001; Kitcher 2001). Yet, at least part of the "developmental noise" appears to be due to chance fluctuations in gene product levels (McAdams and Arkin 1997; Goss and Peccoud 1998). For example, Ozbudak et al. (2002) quantitatively examined whether and how molecular fluctuations within single cells (biochemical noise) may explain the variation of gene expression levels between cells in a genetically identical population (phenotypic noise) in Bacillus subtilis. The authors found that increased translational efficiency is the predominant source of increased phenotypic noise, providing the first direct experimental evidence for a biochemical origin of phenotypic noise.

Microenvironmental phenotypic plasticity is phenotypic sensitivity to microenvironments. It can describe either nongenetic interindividual or intraindividual phenotypic variation. For instance, microenvironmental factors can cause phenotypic variation among clonal individuals of the same genotype within a single macroenvironment. Similarly, microenvironmental factors may cause random deviations from perfect symmetry in individuals of bilaterally symmetric organisms, that is, fluctuating asymmetry (e.g., Palmer and Strobeck 1986, 1992; Debat et al. 2000; Van Dongen and Lens 2000; Hoffmann and Woods 2001; Polak and Starmer 2001). By analogy, microenvironmental canalization is phenotypic insensitivity to microenvironments.

Micro- and macroenvironmental sensitivity and insensitivity are not mutually exclusive: a phenotype can be plastic across macroenvironments but microenvironmentally cana- 
lized, or vice versa (e.g., Schlichting and Pigliucci 1998; Rutherford 2000). For instance, the developmental stability of the petal area of Phlox drummondii depends on the particular macroenvironment of low nutrients, low water, and herbivory (Schlichting and Pigliucci 1998), and changes in fluctuating asymmetry of wing length in $D$. melanogaster and $D$. buzzatii depend on temperature (Imasheva et al. 1997). However, there is often no relationship between micro- and macroenvironmental patterns of phenotypic variation (e.g., Hoffmann and Woods 2001), suggesting that micro- and macroenvironmental canalization are independent. Thus, a single canalizing mechanism will not confer robustness to all kinds of environmental perturbation; many different mechanisms exist by which phenotypes maintain insensitivity to environmental change (Ancel Meyers and Bull 2002; DeVisser et al. 2003; Milton et al. 2003).

\section{GENETIC CANALIZATION OF REACTION NORMS}

Genetic canalization may not only suppress the phenotypic expression of genetic variation of a trait in a given environment but also the expression of genetic variation for phenotypic plasticity. That is, different genotypes can produce the same or a similar phenotype across environments, resulting in the same or a similar level of phenotypic plasticity among genotypes (Wijngaarden et al. 2002). Some authors have used patterns of variation among genotypes across environments to infer genetic canalization (Lewontin 1974b; Stearns 1994). Whether a given bundle of reaction norms is relatively canalized or not is decided by comparison between populations. In a given population, reaction norms may be called on average more or less genetically canalized when compared to another population of reaction norms.

\section{The Molecular Mechanisms of Canalization}

Several molecular mechanisms, which are not mutually exclusive, may contribute to canalization (Wilkins 1997; Gibson and G P Wagner 2000; Hartman et al. 2001; De Visser et al. 2003; Burch and Chao 2004). They include, for example, genetic redundancy, modularity of development, and system-level properties of gene networks and biochemical pathways. Although canalization may be caused by several specific mechanisms, it is useful to retain canalization as a unitary concept because it is likely to play a unitary role in evolutionary processes.

These mechanisms are often reflected as genetic transmission patterns, such as epistasis, genotype by environment interactions, dominance, and pleiotropy, which I discuss below. The most general statement we can make about the causes of canalization is that canalization often is the result of an interaction between the perturbation (e.g., mutation, environment) and the genotype (DeVisser et al. 2003). In fact, as recently shown by Hermisson and G P Wagner (2004), the accumulation of cryptic genetic variation, one of the hallmarks of canalization, is a generic property of systems showing epistasis and genotype by environment interactions.

\section{EPISTASIS}

As I have argued above, canalization is an epistatic phenomenon (Dobzhansky 1971; Gibson 1996; Fenster et al. 1997; G P Wagner et al. 1997, 1998; Rutherford and Lindquist 1998; Hansen and G P Wagner 2001; Hartman et al. 2001; Wade et al. 2001; De Visser et al. 2003; Burch and Chao 2004; Hermisson and G P Wagner 2004).

A well-understood example concerns diazinon insecticide resistance in blowflies, Lucilia cuprina (e.g., Clarke and McKenzie 1987; Davies et al. 1996; overview by Clarke 1997). Resistance to diazinon is essentially conferred by the Rop-1 locus and is correlated with increased levels of fluctuating asymmetry in several bristle traits. Consequently, resistant genotypes have reduced fitness. After resistance had become widespread, however, resistant genotypes were no longer selectively disadvantaged in the absence of the insecticide and had similar fitness as susceptible wildtype genotypes. It was found that selection on the genetic background of resistant genotypes had favored an allele whose spread led to increased levels of environmental canalization (Davies et al. 1996). This allele is an 
allele of the Scalloped wings (Scl) locus, a homolog of the Drosophila gene Notch. Thus, both fluctuating asymmetry as well as environmental canalization are likely to result from an interaction between Rop-1 and Scl.

Another example comes from Gibson et al. (1999) who showed that introgression of homeotic Antennapedia mutations, affecting the antenna-to-leg transformation in $D$. melanogaster, into different wild-type genetic backgrounds substantially increases phenotypic variation, ranging from complete suppression (i.e., canalization) to complete antennal leg formation. Furthermore, using a composite interval mapping method, the authors could demonstrate that one allele $(E(U b x) 3 L)$ accounts for three-quarters of the phenotypic variance for haltere-to-wing margin transformation in $U b x$ mutant flies. This allele, depending on the genetic background, strongly enhances the transformation in $U b x$ flies, but has no obvious effects on wild-type haltere development.

In Caenorhabditis elegans, at least ten genes have been identified that produce mutant vulval phenotypes only in combination with mutations in other genes (Ferguson and Horvitz 1989; Sternberg and Han 1998; Fay and Han 2000). Whereas wild-type C. elegans hermaphrodites have a single vulva, the multivulva phenotypes of certain mutants have three or more vulva-like structures resulting from defects in two functionally redundant pathways. Evidence suggests that the multivulval phenotypes result from interactions between cell-cycle/transcriptional controllers ( $\mathrm{Rb}$ transcriptional regulatory complex) and regulators of vulval development, such as members of the RTK/Ras/Map kinase pathway (e.g., Fay and Han 2000). This case exemplifies synthetic lethality, an epistatic phenomenon (see Hartman et al. 2001): two mutations exhibit synthetic lethality if either of the single mutations (with the full function of the other gene) is viable but mutations in both genes are not. The majority of synthetic lethal relationships occurs among loci acting in the same pathway or process (intrinsic buffering), but some occur among loci in biochemically distinct pathways or processes (extrinsic buffering; Hartman et al. 2001).

A recent study by Elena and Lenski (2001) provides an elegant experimental test for genetic canalization by epistasis in E. coli. The authors examined genetic interactions between random insertion mutations and other mutations by transducing each of twelve insertion mutations into two genetic backgrounds, one ancestral and the other derived. The derived background evolved for 10,000 generations in a laboratory environment. Elena and Lenski (2001) hypothesized that the derived background may have evolved to be better buffered against harmful mutations. In their experiment, there was no compelling evidence for adaptive genetic canalization: while some mutations were less harmful in the derived background (canalization), others showed the opposite pattern, and there was on average no clear trend in either direction. Although the authors identified clear cases of genetic canalization depending on the epistatic interaction present, their data neither demonstrate adaptive canalization nor offer a clear rejection of the hypothesis that canalization evolves by selection (Elena and Lenski 2001).

\section{FUNCTIONAL REDUNDANCY}

Functional redundancy refers to a special kind of epistasis among genes with similar function, causing a mutation at one of the loci to have little or no phenotypic effect (Lerner 1954; Tautz 1992; Nowak et al. 1997; Wilkins 1997; A Wagner 1999; Rutherford 2000; Hartman et al. 2001; De Visser et al. 2003). Thus, functional redundancy in the strict sense means that two or several genes (or pathways) are performing the same (or a similar) function; this redundancy is caused by the presence of paralogous genes resulting from gene duplication among members of the same gene family (see Tautz 1992; Wilkins 1997; McAdams and Arkin 1999; Dover 2000; Hartman et al. 2001; De Visser et al. 2003). Unfortunately, the distinction between canalization and redundancy is often not clear in the literature. For example, some authors also refer to redundancy when functionally unrelated genes are involved in the epistatic interaction (Wilkins 1997; De Visser et al. 2003). Although interactions among unrelated genes may be important for phenotypic 
robustness (e.g., De Visser et al. 2003), functional redundancy as operationally defined by Wilkins (1997) is not different from the general definition of genetic canalization by epistasis. Thus, the term functional redundancy should be reserved for robustness due to the presence of paralogs; also see the above discussion of synthetic lethality.

Functional redundancy seems to be widespread. For instance, in budding yeast, S. cerevisiae, the inactivation of single genes has little phenotypic effect whereas the knockout of all paralogs produces a strong mutant effect (Kataoka et al. 1984). In the case of paralogs of the yeast ras genes, the $C L N$ (cyclin) genes, and the two genes for 3-hydroxy-3-methylglutaryl-coenzyme A, only multiple knockouts have an effect, suggesting that in all three cases paralogs serve as functional backups (Basson et al. 1986; Hadwiger et al. 1989). However, a recent study on the genome of $S$. cerevisiae shows that redundancy may not be important for canalization (A Wagner $2000 \mathrm{~b}$ ). If gene duplications are predominantly responsible for robustness, one would expect a correlation between the similarity of two duplicated genes, both in terms of sequence and temporal expression patterns and the effects of mutations in one of these genes. However, A Wagner (2000b) did not find such an association, suggesting that redundancy due to gene duplication contributes little to mutational robustness. Yet, Gu et al. (2003) demonstrate that null mutations in duplicate genes have a $20 \%$ higher probability of exhibiting only weakly deleterious fitness effects as compared to knockouts in single-copy genes.

\section{PLEIOTROPY}

Pleiotropy, the multiple effects of a single gene or allele on two or more traits, is another potentially important genotypic property bearing on canalization. Since traits are often linked by pleiotropic gene action, pleiotropy may compromise the independence of traits so that it becomes impossible to change a trait without disturbing other traits at the same time (Hansen and Houle 2004). Such pleiotropy, therefore, may limit the evolvability of the focal trait, and it can be argued that pleiotropy thereby contributes to canalization: some traits may become so embedded in development that they lose their evolvability (Stearns 1994; Hansen and Houle 2004). While pleiotropy may impose a constraint for the evolvability of the focal trait, it may also increase the mutational target size and thus improve the evolvability of the trait (Hansen and Houle 2004). While pleiotropy is a common mode of gene action and often emerges as a natural property of networks of transcriptional regulation (Gibson 1996), the relevance of pleiotropy for canalization remains unclear.

\section{DOMINANCE}

Dominance can be viewed as another manifestation of canalization (e.g., Proulx and Phillips 2004 and references therein). It was noticed early on that heterozygotes for mutant and wild-type alleles almost invariably show the wild-type rather than the mutant phenotype; only dominant allele substitutions are expressed. Thus, dominance refers to the concealment of the presence of one allele by the strong phenotypic effects of another allele (Sagaret 1826; Mendel 1866). Dominance may therefore contribute to canalization by masking the effects of deleterious recessive mutations. As has been argued by Wright (1934), recessiveness and dominance are likely to be intrinsic properties of metabolism. Probably most deleterious alleles cause a reduction in enzyme activity. However, if the wild-type allele is more active than required, the rate of reaction is substrate rather than enzyme limited: deleterious mutations appear then as recessive or nearly recessive. For instance, as shown by metabolic control theory, the many gene-controlled steps in a pathway imply that a $50 \%$ reduction in the activity of any one step is often likely to have only minimal effects on the amount of the final product (Wright 1934; Kacser and Burns 1981; Charlesworth 1998).

\section{GENOTYPE BY ENVIRONMENT INTERACTIONS}

Genotype by environment interactions are another mechanism for canalization, conceptually similar to that of epistasis (e.g., Brodie 
2000; Hermisson and G P Wagner 2004). The presence of these interactions implies that different genotypes differ in their degree of plasticity/environmental canalization. Many experiments have established that phenotypic plasticity-and thus, as I argue herein, environmental canalization-is a heritable, evolvable trait (e.g., Waddington 1960; Scheiner and Lyman 1991; see Scheiner 1993 and Roff 1997 for reviews). Recent studies on the genetics of phenotypic plasticity suggest that environment-dependent gene regulation plays a prevailing role in determining a genotype's sensitivity to variation in the environment (Pigliucci 1996; Schlichting and Pigliucci 1998). For instance, environment-dependent alterations of DNA transcription and RNA translation rates are known from the response of flowering plants to light, induced heat-shock proteins in plants, responses of cyanobacteria to sulfur limitation, and temperature-induced sex determination in lizards (Pigliucci 1996). Similarly, Wu (1998) has demonstrated that a few regulatory loci, differing from QTLs (quantitative trait loci) for trait values within environments, are responsible for the environment-dependent control over structural gene expression in Populus trees. This also suggests that epistasis and genotype by environment interactions are not mutually exclusive: the environment interacts with genes that determine the magnitude of the response to the environment, which in turn interact with genes that affect the expression of the trait (epistasis model of phenotypic plasticity; e.g., Scheiner and Lyman 1991).

As shown by several authors, the genetic basis of micro- and macroenvironmental plasticity/canalization is likely to be different (Wu 1998; Debat and David 2001). For instance, Rutherford and Lindquist (1998) have shown that Hsp90 buffers both against genetic and macroenvironmental perturbations (e.g., temperature; see also Queitsch et al. 2002). However, the buffering ability of the hsp 83 locus does not affect microenvironmental variation, as measured by the stochastic effects producing fluctuating asymmetry (Rutherford 2000; Milton et al. 2003). This implies that the mechanisms for the buffering of different kinds of perturbation may not necessarily be identical. For example, canalizing systems such as Hsp90 probably do not generally control both genetic and microenvironmental canalization (Milton et al. 2003). Yet, there are several studies showing that genetic and environmental canalization are correlated and may share the same developmental basis (A Wagner and Stadler 1999; Ancel and Fontana 2000; Burch and Chao 2004).

\section{MODULARITY}

A modular unit of the phenotype is a complex of traits, which collectively serve a primary functional role. Modules are tightly integrated by strong pleiotropic effects and are more or less independent from other such units (G P Wagner 1996; G P Wagner and Altenberg 1996). Modules can be seen at different levels, from base pairs or amino acids to cellular structures or developmental processes (Hartwell et al. 1999; Dover 2000). For a recent discussion of modularity see also Gass and Bolker (2003).

Modularity of development may contribute to canalization (Stearns 1989b; Maynard Smith 1998; Hartwell et al. 1999; Stern 2000). Changes in the organism in one of its parts should not compromise other achievements: independent functions should be coded independently so that the change of one function does not interfere with other optimized functions (G P Wagner and Altenberg 1996). Modularity can be a way to achieve this independence of functions. The significance of modularity for canalization is that perturbing one module does not necessarily perturb the development of the whole organism: the embedding of particular functions into distinct modules allows for phenotypic change by altering connections among the modules while the core function of a given module remains unchanged (G P Wagner and Altenberg 1996; Hartwell 1999; Stern 2000; Schank and Wimsatt 2001). First, if deleterious mutations are highly pleiotropic (but see Stern 2000), then these mutations are likely to have a negative effect on many traits. If gene networks have a modular structure, however, then genetic change in one of the modules does not necessarily influence the others 
(Bonner 1988). Thus, by restricting pleiotropy, modularity allows some modules to continue to function when others change (Schank and Wimsatt 2001). Second, random mutations of a given phenotypic effect are likely to be more deleterious in complex organisms consisting of many traits as compared to simpler organisms with less traits (Fisher 1930; Orr 2000). Fisher (1930) suggested that mutations of small phenotypic effect are more likely to be favorable than mutations with large effects. In a topological model, he showed that the probability of approaching (or deviating from) the fitness optimum is higher (or smaller) if a mutation has a small phenotypic effect than if it has a large phenotypic effect. In the latter case, a mutation is more likely to go beyond the optimum or to deviate from it more strongly than if the mutation has only a small phenotypic effect. For the same intuitive reason, random mutations of a given phenotypic effect are more likely to disrupt a complex than a simple organism (Orr 2000). It would therefore be evolutionarily advantageous to reduce the number of independent traits by bundling them into modules (Orr 2000).

Some of the best examples of modularity concern promoter regions of genes (Dover 2000; Stern 2000). For instance, recent studies of gene regulation, particularly in Drosophila, have demonstrated that the cis-regulatory sequences of many genes are organized into independent modules (e.g., Arnone and Davidson 1997; Stern 2000). These modules are constructed from multiple binding sites for individual transcription factors, and the same module can have several binding sites for the same transcription factor. Thus, the binding sites are redundant both in number and function. While there are many examples of modularity at various levels of biological organization (Hartwell et al. 1999), it is not yet clear how relevant modules are for canalization. This situation may change with the advent of new tools for studying modularity (Magwene 2001).

EMERGENT PROPERTIES OF GENE NETWORKS AND BIOCHEMICAL PATHWAYS

There is increasing evidence that systemlevel properties of gene networks and bio- chemical pathways can lead to robustness against perturbations. Such networks and pathways exhibit emergent properties such as integration of signals across time scales, generation of distinct outputs that depend on input strength and duration, and feedback loops (e.g., Bhalla and Iyengar 1999; McAdams and Arkin 1999). The dynamic stability of these networks often results from connections among the components of the system, such as functional redundancy and feedback regulation.

There are several good examples for the robustness of gene networks and biochemical pathways, both from theoretical and empirical work (De Visser et al. 2003). For instance, enzymes of intermediary metabolism often show saturation kinetics, that is, flux is a concave or (sigmoid) function of enzyme activity (e.g., of the Michaelis-Menten form). If selection favors increased enzyme activity to maximize flux, a point of diminishing returns may be reached where any change in flux results in a disproportionately small change in fitness. Consequently, mutations that affect activity will often result in only minor changes in fitness (Hartl et al. 1985).

As shown by Gibson (1996), thresholddependent transcriptional regulation, the cooperative binding of several transcriptional regulators to a gene promoter, can also lead to canalization. Minor variations in individual parameters can be balanced by variation in other parameters, which allows for the same threshold to be set by different genotypes (Gibson 1996). In a similar vein, A Wagner (1996) modeled sets of transcriptional regulators that form a gene expression network. He demonstrated that the network's stability against deleterious mutations increases under stabilizing selection so that the fraction of mutations causing changes in gene expression patterns is substantially reduced, regardless of the network structure and the mode of reproduction.

Barkai and Leibler (1997) showed that simple biochemical networks of bacterial chemotaxis are robust against perturbations due to the network's connectivity. Similarly, theory developed by Frank (1999) shows that the more highly connected a Boolean regulatory network, the smaller the effect of muta- 
tional perturbation. Lenski et al. (1999) constructed digital organisms and showed that organisms with a more complex genetic architecture are more robust than simple ones with respect to the average effects of mutations.

As found by Little et al. (1999), the behavior of the phage Lambda gene regulatory circuit exhibits robustness of transcriptional regulation. The phage can switch between two states: lysogeny (integration of the viral genome into the host's genome without destroying the host cell) and lysis (viral replication and destruction of the host cell). This switch involves a promoter lying between two autoregulatory genes, which have different affinities for different modular binding sites in the promoter. The authors experimentally altered the normal binding pattern such that the binding sites for the two regulatory proteins had identical sequences. Despite these changes, the mutations in the two regulatory sites showed the same qualitative in vivo pattern of gene expression as the wild-type. Similarly, using a synthetic gene circuit in E. coli, Becskei and Serrano (2000) demonstrated that autoregulatory negative feedback loops can dramatically reduce variation in gene expression. Wilke et al. (2001) demonstrated that populations of digital organisms that have evolved under high mutation rates tend to be more robust to mutation as compared to populations that have evolved in identical environments at low mutation rates. At high mutation rates, mutationally more robust populations prevailed in competition with less robust populations.

Interestingly, Siegal and Bergman (2002) showed that developmental processes, modeled as interaction networks of transcriptional regulators, can produce canalization, even without selection towards an optimum. Finally, using both computer simulations of gene networks and an analysis of gene-knockout data from $S$. cerevisiae, Bergman and Siegel (2003) presented evidence that suggests that most genes release phenotypic variation when their function is compromised (but see, for example, Thatcher et al. 1998). Furthermore, the availability of such loss-of-function mutations can-at least theoretically-accelerate adaptation to a new optimum pheno- type, and this effect does not seem to require that the mutations are conditional on the environment.

\section{The Evolution of Canalization}

\section{ADAPTIVE AND NONADAPTIVE ORIGINS OF CANALIZATION}

The evolution of canalization is difficult to address because there are many alternatives that are difficult to distinguish empirically. In principle, canalization could result from adaptive evolution (e.g., Schmalhausen 1949; Waddington 1957; Layzer 1980; Gavrilets and Hastings 1994; G P Wagner et al. 1997; Rice 1998; Kawecki 2000) or as an emergent property of complex gene and trait networks (e.g., Kauffman 1993; Barkai and Leibler 1997). Thus, following De Visser et al. (2003), we may distinguish three origins of canalization: (1) adaptive; (2) intrinsic; and (3) congruent. An adaptive origin implies that canalization evolves because the insensitivity to perturbations increases fitness; an intrinsic origin implies that canalization arises as a nonadaptive correlated byproduct of functional trait architecture; a congruent origin implies that genetic canalization evolves as a correlated response to selection for environmental canalization.

As I have outlined above, there may not be a single specific mechanism for canalization. On the other hand, if specific single mechanisms do exist, the evolution of canalization will be much simpler to address-both theoretically and empirically-than if there are multiple mechanisms for canalization, some of them having evolved for reasons other than canalization. Thus, there are several important questions to which we do not yet have clear answers. Here I concentrate on the case in which canalization evolves by natural selection, as assumed by Waddington and in most theoretical treatments of the evolution of canalization (e.g., Gavrilets and Hastings 1994; G P Wagner et al. 1997; Kawecki 2000).

\section{MODELS FOR THE EVOLUTION OF CANALIZATION}

It is thought that canalization holds phenotypes close to their fitness optimum. Consequently, it is expected that stabilizing selec- 
tion favors the evolution of both genetic and environmental canalization (e.g., Schmalhausen 1949; Waddington 1957; Rendel 1967; Layzer 1980; Stearns and Kawecki 1994; A Wagner 1996; G P Wagner et al. 1997; Rice 1998).

First, and most generally, canalization can in principle evolve whenever there is a nonlinear relationship between traits or between loci (e.g., A Wagner 1996; Rice 1998, 2000; see the phenotypic landscape model above). Second, traits are often connected to fitness nonlinearily. If the relationship between fitness and a trait is convex, then reducing the phenotypic variance of the trait will increase fitness. If the relationship is concave, however, then increasing the variance will increase fitness (e.g., Stearns 2000). This principle, discovered by the Swiss mathematician Daniel Bernoulli in 1738 (Stearns 2000), is important in economics and has been applied to evolutionary biology by Stephens and Krebs (1986) and Real and Ellner (1992). As shown by theoretical work, canalization can evolve when fitness functions are convex (i.e., stabilizing selection; Lande 1980; Layzer 1980; Rice 1998; Kawecki 2000). Third, convexity of the fitness function is not necessarily synonymous with selection for canalization: both randomly and periodically fluctuating linear as well as Gaussian selection regimes can select for genetic canalization (Kawecki 2000). Fourth, directional selection is thought to select against canalization (Thoday 1958; Layzer 1980; G P Wagner et al. 1997): directional selection can favor modifiers that increase the phenotypic expression of genetic variation. Fifth, new work shows that the maximum strength of selection for environmental or genetic canalization is generally a function of the fitness load imposed by the environmental or genetic perturbation (Hermisson et al. 2002; Proulx and Phillips 2004).

The evolution of canalization has usually been modeled assuming canalizing modifiers. Canalizing modifiers are alleles that reduce the phenotypic effect of one or several other alleles (G P Wagner et al. 1997). Models of canalization often assume that a trait is affected by two kinds of loci: structural loci that have a direct effect on the trait and epi- static modifier loci that reduce the phenotypic expression of the structural loci. Simple models therefore rely on the somewhat unrealistic assumption that only one or a few genes or alleles have strong epistatic effects whereas most genes or alleles have only additive genetic effects (G P Wagner et al. 1997). Consequently, more general theoretical models of canalization have been developed in which loci both have direct effects on the trait and modify the phenotypic expression of other loci (G P Wagner et al. 1997; Rice 1998).

\section{SELECTION FOR GENETIC CANALIZATION}

Stabilizing selection reduces genetic variance by driving the segregating loci underlying the trait to fixation. However, even under stabilizing selection, deviations from the fitness optimum may be frequent because, if epistasis and pleiotropy are common, selection cannot eliminate all genetic variance for the trait. Genetic variance that causes individuals in the population to deviate from their fitness optimum is thus thought to be the driving force for the evolution of genetic canalization: the more genetic variance, the stronger the selection for canalization (G P Wagner et al. 1997). All factors contributing to genetic variance-large population size, high mutation rate, and a large number of loci-favor the evolution of genetic canalization. Under mutation-selection balance, selection cannot completely eliminate deleterious alleles because they occur continuously and recurrently, and canalization may therefore be selected. However, the conditions under which genetic canalization can evolve under mutation-selection are rather restrictive (G P Wagner et al. 1997). The strength of stabilizing selection may have counteracting effects. On the one hand, increasing the strength of stabilizing selection favors selection for genetic canalization. On the other hand, as the strength of selection increases more and more (i.e., selection becomes purifying), stabilizing selection eliminates genetic variance to a point where canalization cannot evolve anymore (G P Wagner et al. 1997). Furthermore, if one assumes that alleles with canalizing effects have direct (pleiotropic) effects on the trait, any direct effect will be dele- 
terious and selected against whereas canalizing effects will be selected for. Under strong stabilizing selection, genetic canalization may not evolve because selection for canalizing effects cannot override selection against direct effects (G P Wagner et al. 1997). Thus, stabilizing selection may favor genetic canalization only at low to moderate strengths of selection (G P Wagner et al. 1997). Interestingly, recent work by Hermisson et al. (2003) shows that stabilizing selection does not typically lead to genetic canalization of the trait. In fact, the conditions for the evolution of genetic canalization of traits are even more restrictive than the work by G P Wagner et al. (1997) suggests. Instead, canalization may evolve at the gene level; such genic canalization refers to the buffering of mutational effects of certain subunits or groups of genes (Hermisson et al. 2003). Only genes with high mutation rates will primarily be buffered, however, and the genic buffering may often occur at the expense of decanalization of other genes (Hermisson et al. 2003). Also see De Visser et al. (2003) for a discussion of the effects of mutation rates, modes of reproduction, strength of selection, and population size on the evolution of genetic canalization.

Three points are particularly important here. First, selection for genetic canalization is usually weak, that is, selection coefficients are small (G P Wagner et al. 1997; Kawecki 2000; Proulx and Phillips 2004). However, as shown by Proulx and Phillips (2004), the strength of selection for genetic canalization may depend on the size of the genetic network: selection for canalization is typically stronger in larger genetic networks because large networks exhibit a higher mutational load than small networks. Second, different assumptions about the maintenance of genetic variance may considerably change the theoretical predictions made by G P Wagner et al. (1997). Forces other than mutationselection balance can account for the maintenance of genetic polymorphism (e.g., Roff 1997), such as environmental heterogeneity (Felsenstein 1976; Hedrick 1986), pleiotropy (Rose 1982), epistasis (Gimelfarb 1989), and heterosis. These processes can maintain relatively large amounts of genetic variance in natural populations (e.g., Turelli 1988) and may be a driving force for the evolution of canalization. Thus, mechanisms for the maintenance of genetic variation other than mutation-selection balance may facilitate selection for genetic canalization. Third, in the model by G P Wagner et al. (1997), environmental canalization (see below) evolves rather easily as compared to genetic canalization (also see Proulx and Phillips 2004). There are several lines of evidence suggesting that patterns of genetic and environmental canalization may be correlated (Ancel and Fontana 2000; Burch and Chao 2004): traits that are under stronger stabilizing selection seem to be both environmentally and genetically more canalized than traits under weaker selection (Stearns and Kawecki 1994; Stearns et al. 1995). If this is the case, then the evolution of genetic canalization could be a correlated response to the evolution of environmental canalization (G P Wagner et al. 1997). For instance, A Wagner and Stadler (1999) demonstrated that the secondary structure of a viral RNA genome is more stable than a randomly chosen sequence that folds into the same structure, suggesting that the virus has evolved to a state of increased mutational stability. In this case, the mutational stability (genetic canalization) may be a side effect of selection for increased thermodynamic stability (environmental canalization). Such a mechanism has also been shown to be at work in a model of the evolutionary dynamics of RNA secondary structure (Ancel and Fontana 2000).

Theoreticians have also studied the evolution of particular mechanisms of genetic canalization, for example, genetic redundancy. One problem with the theory of the evolution of genetic redundancy is that genes encoding functional proteins must be under selection pressure: if a gene is truly redundant, then it is not protected against the accumulation of deleterious mutations (Nowak et al. 1997). Consequently, redundancy may not be evolutionarily stable. However, as shown by Nowak et al. (1997), evolutionarily stable redundancy can evolve by selection in several simple models. The models assume that genes are initially neither redundant nor pleiotropic: each gene performs a single unique 
function. Then, spontaneously arising mutations that lead to a functional overlap can be favored by selection. These mutations can become fixed, and redundancy can become evolutionarily stable if random mutations occurring in the genome are more likely to destroy all functions of a gene rather than to destroy just one function while leaving others unaffected. The final genetic configurations often consist of complex genetic networks, exhibiting both redundancy and pleiotropy: each function is performed by several genes, and each gene performs several functions (Nowak et al. 1997). Similarly, A Wagner (1999) has found that selection can maintain as well as increase redundancy among genes in a population provided that mutations generate sufficient variation in redundancy and that populations are large. Selection acts through the decreased number of offspring with deleterious mutations that individuals with redundant genes generate (A Wagner 1999). As shown by A Wagner (2000a), however, the average effects of deleterious mutations on fitness do not greatly influence the evolution of functional overlap and vice versa: the extent of functional overlap maintained in a population is independent both of the mutation rate and the average effects of mutations. Thus, overlapping gene functions may not be maintained because they confer a higher mean fitness by reducing the impact of deleterious mutations. This implies that the maintenance of functional overlap may not be an adaptive case of canalization.

\section{SELECTION FOR ENVIRONMENTAL} CANALIZATION

Generally, since environmental canalization confers insensitivity to environmental change, it will only be selected for if the optimum phenotype is the same across environments. In contrast, if the optimal phenotype depends critically on the particular environment, phenotypic plasticity should be favored by selection (De Visser et al. 2003). In contrast to genetic perturbations, environmental perturbations often cause strong selection for canalization (e.g., Proulx and Phillips 2004).

Models by Gavrilets and Hastings (1994) and G P Wagner et al. (1997) have addressed selection for canalization against microenvironmental perturbations. Their analyses suggest that stabilizing selection will favor the evolution of canalization against developmental noise. Under stabilizing selection, reduced microenvironmental variation is always associated with increased mean fitness: the stronger the stabilizing selection and the larger the reduction of microenvironmental variation through the canalizing effects of a modifier, the stronger the selection for canalization (G P Wagner et al. 1997). Microenvironmental variation is never reduced to zero, however, suggesting that there is a limit to microenvironmental canalization ( $\mathrm{G} P$ Wagner et al. 1997). This may be due to canalizing modifiers having negative direct (pleiotropic) effects on the trait (G P Wagner et al. 1997). Furthermore, empirical evidence suggests that it is possible to select for increased microenvironmental canalization. For example, it is possible to select for decreased fluctuating asymmetry in scutellar bristle number in D. melanogaster (see Scharloo 1988, 1991).

Several examples suggest that macroenvironmental canalization (i.e., reduced plasticity) can be selected. For instance, Waddington (1960) applied a family selection regime to select on the facet number of the Bar mutant in D. melanogaster, in which the offspring of pair-matings were divided between two treatments, one at $18^{\circ} \mathrm{C}$ and one at $25^{\circ} \mathrm{C}$. The facet number is greater at lower than at higher temperatures. Selection was made on the basis of differences among family means. Waddington's experiment yielded fast progress: in the unselected Bar stock, the facet number at $18^{\circ} \mathrm{C}$ was initially three times higher than at $25^{\circ} \mathrm{C}$. However, after six generations of selection, the difference had been reduced to about $10 \%$. At this point, the phenotypes at both temperatures were intermediate between those seen at the start of the experiment.

Similarly, Scheiner and Lyman (1991) selected on phenotypic plasticity of thorax size in response to temperature in $D$. melanogaster. In the lines selected for decreased plasticity, a selection limit of zero plasticity was reached, but additive genetic variance for plasticity still existed. The authors succeeded 
in decreasing plasticity so that flies raised at $19^{\circ} \mathrm{C}$ were on average the same size as flies raised at $25^{\circ} \mathrm{C}$. This pattern was irreversible since it was not possible to select for increased plasticity again. Although a selection limit was reached, there were still substantial amounts of additive genetic variance as measured by narrow-sense heritabilities based on sib correlations after 16 generations of selection. The authors explain this apparent contradiction by suggesting that the plasticity of the trait is determined by loci separate from those determining the mean of the trait.

While Waddington (1960) and Scheiner and Lyman (1991) managed to select for increased macroenvironmental canalization (i.e., less steep reaction norms), a recent selection experiment failed to change the shape and the slope of the reaction norms for eyespot size in the butterfly Bicyclus anynana (Wijngaarden et al. 2002). The authors only managed to change the elevation of a bundle of reaction norms. This may suggest that the bundle is canalized so as to exhibit the same overall shape and slope by some unknown mechanism, for instance by hormonal regulation. Alternatively, the shape and slope of the reaction norms may not be controlled by the same loci as the reaction norm's elevation. Thus, the result may simply be explained by a lack of standing genetic variation for the shape and slope of the reaction norms.

\section{CANALIZING SELECTION}

The classic view of selection assumes that selection eliminates additive genetic variance by reducing the number of segregating alleles, that is, by driving their frequencies to zero or one. In contrast, canalizing selection (Waddington 1957) selects for genetic mechanisms that suppress the phenotypic expression of genetic variance. It thereby retains the genetic potential for phenotypic change. The difference between the two forms of selection can be illustrated by considering the additive genetic variance of a trait determined by $n$ loci in linkage equilibrium:

$$
\mathrm{V}_{\mathrm{A}}=2 \sum_{i=1}^{n} \mathrm{a}_{\mathrm{i}}^{2} \mathrm{p}_{\mathrm{i}}\left(1-\mathrm{p}_{\mathrm{i}}\right)
$$

where $p_{i}$ and $q_{i}=1-p_{i}$ represent the allele frequencies at locus $i$ and $\mathrm{a}_{\mathrm{i}}$ is the effect of the two alleles segregating at locus $i$. There are two ways to decrease genetic variance: (i) either by driving $p_{i}$ or $q_{i}$ to zero or to one, thus eliminating genetic variance; or (ii) by decreasing the effects of the segregating alleles, $\mathrm{a}_{\mathrm{i}}$. Canalizing selection does the latter; it reduces the magnitude of allelic effects (e.g., Stearns and Kawecki 1994; G P Wagner et al. 1997).

Selection that does not alter the mean of a trait but reduces its variance is commonly called stabilizing selection. Subsequently, it has been suggested that canalizing selection refers to a special kind of stabilizing selection (Schmalhausen 1949; Waddington 1957; Stearns and Kawecki 1994; G P Wagner et al. 1997). However, canalization can evolve not only by stabilizing selection but also by fluctuating linear or Gaussian selection (Kawecki 2000). Selection thus qualifies as canalizing selection, no matter what the fitness function looks like, if it favors the suppression of the phenotypic expression of genetic variation.

In principle, selection can therefore reduce phenotypic variation by reducing genetic variance, its phenotypic expression, or both. In reality, it is most likely that the two types of selection are not completely independent and that they occur simultaneously (Stearns and Kawecki 1994). For most episodes of selection in natural or experimental populations, we do not know whether phenotypic variation decreased because alleles were fixed or eliminated or whether the phenotypic effects of segregating alleles were masked by selection for canalizing mechanisms.

\section{The Evolutionary Significance of Canalization}

\section{CANALIZATION AND FITNESS SENSITIVITY}

Any developmental mechanism that canalizes a trait by keeping it close to its fitness optimum should be favored by selection (Rendel 1967). Consequently, traits that are under strong stabilizing selection should be more canalized than traits under weak selection (but see G P Wagner et al. 1997). This is exactly what Stearns and Kawecki (1994) and Stearns et al. (1995) found in experiments on D. melanogaster. The lines they used differed 
in the position of a P-element insert or in genetic background; flies within lines were genetically nearly identical. Genetic canalization was measured as the inverse of the coefficient of variation among lines; microenvironmental canalization was measured as the inverse of the coefficient of variation among replicates within lines. For both genetic and environmental canalization, the canalization of traits increased with their impact on fitness and did not depend on genetic differences among lines. The genetic and environmental coefficients of variation were positively correlated. This suggests that (i) there is differential genetic and environmental canalization of fitness components and (ii) the same developmental mechanisms may buffer the phenotype against genetic and microenvironmental perturbation. However, these results and the underlying assumptions were questioned by Houle (1998), who reanalyzed data on genetic variances in D. melanogaster from the literature. He found evidence that the mutational variance of traits was likely to be influenced by the mutational target size (i.e., traits with a complex functional architecture are larger targets for mutation) and by the timing of trait expression (i.e., traits expressed later in life inherit variation from earlier stages). Canalization did not explain differences in the genetic variances of traits (Houle 1998), however. Thus, Houle (1998) concluded that canalization is unlikely to play a dominant role in determining variation in mutational and standing genetic variances (see also Hermisson et al. 2003).

\section{CANALIZATION AND RECOMBINATIONAL LOAD}

Canalization may reduce the problem of recombinational load. Whereas in asexual organisms mutations enter the population sequentially, progeny of sexual organisms face the problem of genetic recombination (e.g., Barton and Charlesworth 1998). Recombination can induce a genetic load if the trait is under stabilizing selection and the mean phenotype has approached the fitness optimum. Under recombination, newly produced phenotypes will often deviate from the optimum. However, phenotypes have to be pro- duced in reliable, functional trait combinations despite genetic recombination. Genetic canalization may be one way of stabilizing the development of sexual organisms facing the problem of recombination (e.g., Stearns 1994; G P Wagner and Altenberg 1996). We do not know enough yet to check the validity of this hypothesis. However, theory suggests that there are similarities between the selection regimes that favor genetic canalization and the conditions that favor low recombination rates (see discussion in Kawecki 2000). Both genetic canalization (e.g., G P Wagner et al. 1997) and low recombination rates (e.g., Charlesworth 1993) can be favored by stabilizing selection.

\section{CANALIZATION AND CONSTRAINT}

Canalization may constrain phenotypic evolution, that is, prevent (or slow down) the attainment of fitness optima by an evolving population (Maynard Smith et al. 1985). Since the response to selection is proportional to the amount of additive genetic variance expressed in the phenotype, canalization can potentially reduce a trait's capacity for evolution by reducing its level of expressed variation. Although mutations continually occur in the genes affecting all traits, some traits are invariant within clades. Stearns (1994) suggested that canalization may be responsible for this invariance by decoupling variation in traits from variation in genes. Under this view, canalization may lead to macroevolutionary patterns (e.g., traits fixed within lineages) that suggest phylogenetic and developmental constraints. Thus, short-term canalization may temporarily constrain phenotypic evolution, and longterm canalization may lead to character stasis (Charlesworth et al. 1982; Stearns 1982; Maynard Smith et al. 1985; Scharloo 1987; Bradshaw 1991; Stearns 1994).

\section{CANALIZATION AND EVOLVABILITY}

Canalization, followed by decanalization, may increase evolvability, the ability of a population to respond to selection. The evolvability of a trait is proportional to the amount of heritable genetic variance, a result following from Fisher's fundamental theorem 
of selection (Houle 1992). Canalization can increase evolvability because it allows the accumulation of genetic variation not "seen" by selection. The cryptic variation allowed to build up by canalization can be released under decanalizing conditions, and selection on the trait can change the frequency of the previously cryptic alleles (Levin 1970; Agur and Kerszberg 1987). Experimental examples from D. melanogaster (Rutherford and Lindquist 1998; see above) and budding yeast, $S$. cerevisiae, show that canalization can increase evolvability. In budding yeast, a modified protein, the prion $\left[\mathrm{PSI}^{+}\right]$, is an epigenetic modifier of the fidelity of translation termination. It provides the means to uncover hidden genetic variation for growth rates and to produce new heritable growth rate phenotypes across diverse environments (True and Lindquist 2000; True et al. 2004). However, it is questionable whether these systems have adaptively evolved to a state of increased evolvability (Partridge and Barton 2000).

\section{CANALIZATION AND GENETIC ASSIMILATION}

A particular way in which canalization may increase evolvability is genetic assimilation. Genetic assimilation is defined as the process by which a phenotype originally produced only in response to some environmental change becomes stably expressed independently of the evoking environmental effect (Waddington 1953; Pigliucci and Murren 2003). It requires that: (i) a population is originally genetically polymorphic for a particular polygenic threshold trait, but that the alleles for this phenotype are not expressed because of canalization; (ii) an environmental stimulus or allele substitution decanalizes the expression of these alleles; and (iii) selection alters the frequency of these alleles to the point where the phenotype appears in the absence of the environmental stimulus or allele substitution (Stern 1958; Waddington 1961; Scharloo 1991; Hall 1999; Gilbert 2000).

Genetic assimilation may lead to rapid phenotypic divergence and may-at least theoretically-facilitate adaptation to novel environments (Levin 1970; Eshel and Matessi 1998) and speciation (Pál and Miklós 1999).
There are several experimental examples of genetic assimilation (Scharloo 1991; Rutherford and Lindquist 1998; True et al. 2004). For instance, Waddington (1953) has studied genetic assimilation of the cross-veinless phenotype $(c v l)$, a defect in wing venation pattern in D. melanogaster. Waddington started a selection experiment with a wild-type base population in which no col flies appeared when raised at $25^{\circ} \mathrm{C}$. However, when pupae were given a heat shock of 4 hours at $40^{\circ} \mathrm{C}$ at 17 to 23 hours after puparium formation, some proportion of the flies showed the col phenotype. Waddington created two selection lines by treating pupae in every generation with heat shock and by selecting flies as parents for the next generation which either showed the col phenotype after heat shock ("upward" selection line) or not ("downward" selection line). The frequency of the col phenotype increased in the upward treatment and decreased in the downward treatment. After 13 generations, the difference in the percentage of the $c v l$ phenotype between the two treatments had become about $60 \%$. Furthermore, Waddington examined in every generation a large number of flies from untreated pupae of the upward selection regime. Among these untreated control flies, no $c v l$ individuals were found until generation 14. At generation 16 the frequency of $c v l$ had raised to $1-2 \%$, and Waddington set up paired matings between such $c v l$ individuals. From these flies, he created four selection lines and obtained a high proportion of $c v l$ phenotypes at $25^{\circ} \mathrm{C}$ without any heat shock. Thus, Waddington had "genetically assimilated" an environmentally induced phenotype.

Another example comes from Gibson and Hogness (1996), who repeated an experiment of Waddington's (1956) by selecting for differential sensitivity to the induction of bithorax phenocopies by ether vapor (see also Ho et al. 1983a,b,c). Bithorax phenocopies resemble bithorax mutants, which have two pairs of wings. Using molecular genetics, they demonstrated that the differential phenotypic expression of the ether-induced bithorax phenotype can be mostly attributed to genetic polymorphism at the Ubx (Ultrabithorax) locus. Loss of expression of Ubx pro- 
tein in the third thoracic imaginal discs correlated with increased sensitivity to ether. This shows that there is heritable genetic variation for the propensity to exhibit etherinduced bithorax phenotypes, which can be selected upon. Selection will then lead eventually to the loss of Ubx expression, resulting in a high proportion of bithorax phenotypes, which are stably expressed in the absence of ether.

Although Waddington (1953) suggested that changes in canalization may account for assimiliation, he did not propose an explicit mechanistic model. However, most assimilation experiments are consistent with threshold models of trait expression (Scharloo 1991; Hall 1999); these models assume that the trait under consideration is a polygenic threshold trait. These assumptions have been confirmed in several experiments (Rutherford and Lindquist 1998). In these models, a decanalizing perturbation can move the population outside of a zone of canalization in which the variation is masked. Consequently, previously hidden genetic variation for the trait becomes exposed, making selection more effective. Eventually, selection will shift the trait into a new zone of canalization where the new phenotype becomes stably expressed in the absence of the enabling stimulus.

\section{CONFLICTING EVOLUTIONARY ROLES OF CANALIZATION?}

Canalization may have important implications for both micro- and macroevolution: optimal phenotypic design, rapid phenotypic divergence and speciation, stasis and constraints. However, the relative importance of canalization for these different phenomena is not yet clear (De Visser et al. 2003). For instance, whether genetic canalization is adaptive, that is, leads to an increase in the mean fitness of a population, crucially depends on the mechanism maintaining genetic variation (G P Wagner et al. 1997). Theory shows that in the case of mutationselection balance, for example, there is no fitness benefit from genetic canalization because under mutation-selection balance the mean fitness is independent of the aver- age effect of the mutations-a consequence of the Haldane-Muller principle (G P Wagner et al. 1997). Furthermore, the frequent observation that mutants or environmentally induced phenotypes are more variable than the wild-type does not imply that the wild-type is adapted to be more canalized (Hansen and G P Wagner 2001). Similarly, whether canalization has a constraining effect, by reducing the response to selection, will depend on the stability of the canalizing mechanisms and on the frequency and magnitude of decanalizing perturbations. For instance, directional selection on a trait can favor decanalization (e.g., Layzer 1980). However, theory suggests that even strong directional selection is likely to be rather slow in decanalizing, and short periods of directional selection are unlikely to decanalize a well-canalized trait (G P Wagner et al. 1997). The temporary breakdown of canalizing mechanisms may result in alternating phases of canalization and decanalization, leading to punctuated phenotypic dynamics (e.g., Agur and Kerszberg 1987).

\section{Detecting and Measuring Canalization \\ STANDARD METHODS}

There are essentially two approaches to detect and measure canalization (Gibson and G P Wagner 2000; De Visser et al. 2003; also see Gibson and Dworkin 2004 for methods of measuring cryptic genetic variation), both involving genetic/environmental perturbation of a reference population. Genetic perturbation can be achieved, for instance, by P-element insertion (Stearns and Kawecki 1994), mutation accumulation, or introgression of mutations into isogenic lines (Moreno 1994; Gibson et al. 1999). Environmental perturbation can be achieved, for example, by heat shock, treatment with specific inhibitors of gene action (geldanamycin in the case of the $h s p 83$ locus), or chemicals such as ether vapor (Scharloo 1991; examples above).

First, genetic canalization can be detected most clearly by comparing mutational variances among different lines in a mutation accumulation experiment. Starting with an isogenic line, the phenotypic variance is 
entirely due to environmental effects. Over time, however, sublines founded from the isogenic line will accumulate mutations so that the variation among sublines as well as the average within-line phenotypic variance will increase over time. If the rate of increase of variation in one trait is smaller than in another, the trait is canalized (Gibson and G P Wagner 2000). However, this approach has several drawbacks including large measurement errors, a bias due to the use of isogenic lines, and other confounding factors affecting variation (see Gibson and G P Wagner 2000).

Second, one can check for canalization by measuring the change in phenotypic variance among lines that are perturbed and not perturbed (Scharloo 1991 for some classic examples). For instance, one can compare two sets of inbred sublines originating from two different lines. Then one compares the two sets of sublines when perturbed and not perturbed. When unperturbed, the sublines in both sets are likely to exhibit some variation in mean phenotype. If the two sets are now perturbed, the overall mean phenotype is likely to change in both sets. If the variation in mean phenotypes among sublines in a given set changes less or not at all as compared to the other set, then this set is more canalized than the other. Such an approach has been used for instance to examine the effects of introgression of mutations into welldefined genetic backgrounds (e.g., Moreno 1994; Gibson and van Helden 1997; Elena and Lenski 2001). Phenotypic variances of the same trait or of different traits can then be compared by using coefficients of variation, that is, variances standardized by the mean or the square of the mean (e.g., Houle 1992; Stearns et al. 1995; Houle 1998).

\section{MOLECULAR METHODS}

The recent advent of molecular tools makes it now possible to address questions about the molecular mechanisms of canalization directly (e.g., Gibson and Dworkin 2004). For instance, the genetic control of canalization by short chromosome segments can be studied by using DNA-based markers (Routman and Cheverud 1994; Jansen 1995).
With such methods it is possible to determine the number, location, and magnitude of effects of quantitative trait loci (QTLs). QTL mapping has helped to define genotype by environment interactions and can also be used to define epistatic effects (Cheverud and Routman 1995). Thus, the method in principle allows one to identify genes involved in buffering by identifying QTLs for the trait, which is canalized. Then, one can ask whether these loci act additively or whether they interact epistatically. For instance, QTL mapping has been successfully applied in mice where QTLs affecting fluctuating asymmetry in skull morphology have been identified (Leamy et al. 1998). Similarly, recombination mapping protocols have been used to identify Notch as a candidate gene responsible for the reduction of fluctuating asymmetry in blowflies (Davies et al. 1996).

\section{PROBLEMS WITH DETECTING CANALIZATION: A CAUTIONARY NOTE}

This review has discussed and highlighted the potential evolutionary importance of canalization and cryptic variation. Canalization can be defined as reduced sensitivity to genetic or environmental perturbation, and its presence can be demonstrated by showing a release of cryptic variation caused by a decanalizing perturbation. Thus, it is rather straightforward to show that canalization occurs as a consequence of epistasis or genotype by environment interactions (Elena and Lenski 2001), and several examples of canalization now exist (Scharloo 1991 for a review; Rutherford and Lindquist 1998 for an example). Furthermore, the recent development of measurement-theoretical representations of epistasis will facilitate the analysis and interpretation of experimental tests of genetic canalization (Hansen and G P Wagner 2001).

Yet, despite the potentially important evolutionary implications of canalization, the concept remains problematic. Recently, Hermisson and G P Wagner (2004) have challenged the concept by demonstrating that cryptic variation can build up even in the absence of canalization of the wild-type. As a consequence, classic experiments showing 
the release of cryptic variation may not provide sufficent evidence that the hidden variation was held in check by canalization. Even if specific canalizing patterns do exist and cause the buildup of cryptic variation, showing that canalization is adaptive is extremely difficult. Most, if not all, experiments (Scharloo 1991) do not clearly address whether (or fail to demonstrate that) canalization is adaptive. Thus, devising novel approaches to demonstrate unambiguous cases of canalization will be of crucial importance (Hansen and G P Wagner 2001; Hermisson and G P Wagner 2004). Yet, the phenomenon of cryptic variation seems to be a real and generic property of populations with epistasis and genotype by environment interactions (Gibson and Dworkin 2004; Hermisson and G P Wagner 2004). Future work needs to address whether the phenomenon of cryptic variation, its buildup and release, is evolutionarily important, independent of whether it is caused by canalizing mechanisms or not (Gibson and Dworkin 2004; Hermisson and G P Wagner 2004).

\section{Summary ANd Conclusions}

I have emphasized several points in this review. (1) Canalization is the relative phenotypic insensitivity of a genotype to genetic or environmental change. A canalized genotype produces the same (or a similar) phenotype in different genetic backgrounds (genetic canalization) or in different environments (environmental canalization). (2) Concepts such as homeostasis and buffering are essentially synonymous with canalization. (3) Genetic canalization is a special kind of epistasis. (4) Environmental canalization and phenotypic plasticity are two aspects of the same phenomenon. (5) Genetic canalization and phenotypic plasticity are not mutually exclusive. (6) Canalization and phenotypic plasticity can be generalized using the notion of variability, that is, the capacity to respond to genetic and environmental change. They can be modeled using phenotypic landscapes. (7) Genetic canalization results in the accumulation of phenotypically cryptic genetic variation. (8) On the one hand, canalization may increase evolvability and lead to phenotypic diversification after a decanaliz- ing event. On the other hand, canalization may, at least temporarily, constrain phenotypic evolution. (9) Several molecular mechanisms may lead to canalization, among them genetic redundancy, modularity, and systemlevel properties of gene networks and biochemical pathways. These mechanisms are not mutually exclusive; they are often reflected in properties such as epistasis, genotype by environment interactions, and pleiotropy. Generally, it can be stated that canalization is an interactive and context-dependent phenomenon. (10) Different forms of selection can favor canalization, but the requirements for the evolution of canalization are usually rather restrictive. (11) Canalizing selection favors genetic mechanisms that result in the suppression of genetic variation at the level of the phenotype. (12) Although there are several methods to detect canalization, there are still serious problems with unambiguously demonstrating canalization, particularly adaptive canalization.

Despite the recent progress reviewed here, there is not yet a consensus in the field on some of the most fundamental problems. Two major unresolved problems are about (i) how canalization should be measured, and (ii) whether canalization is an adaptive phenomenon or rather an intrinsic property of gene networks and biochemical pathways. We have many patterns and hypotheses but little solid data. Unraveling the developmental and evolutionary basis of canalization will be an important task for evolutionary developmental biology and evolutionary genetics. Most importantly, however, unambiguously demonstrating that canalization is more than a beautiful idea remains a major challenge for future research.

\section{ACKNOWLEDGMENTS}

I am very grateful to Steve Stearns for encouragement and inspiration and to Nicole Vouilloz for all her insight, help, and constructive critique. I also thank Christian Braendle, Dieter Ebert, Joachim Hermisson, Tad Kawecki, Benedikt Schmidt, Steve Stearns, Günter Wagner, and two anonymous referees for helpful comments on the manuscript. Santiago Elena, Richard Lenski, and Claus Wilke kindly provided me with preprints of their work. My work was supported by the Swiss Nationalfonds (grant to T J Kawecki), the Roche Research Foundation, and the Swiss Study Foundation. 


\section{REFERENCES}

Agur Z, Kerszberg M. 1987. The emergence of phenotypic novelties through progressive genetic change. American Naturalist 129:862-875.

Ancel L W, Fontana W. 2000. Plasticity, evolvability, and modularity in RNA. Journal of Experimental Zoology Part B: Molecular and Developmental Evolutions 288(3):242-283.

Ancel Meyers L, Bull J J. 2002. Fighting change with change: adaptive variation in an uncertain world. Trends in Ecology and Evolution 17:551-557.

Arnone M I, Davidson E H. 1997. The hardwiring of development: organization and function of genomic regulatory systems. Development 124:18511864.

Barkai N, Leibler S. 1997. Robustness in simple biochemical networks. Nature 387:913-917.

Barton N H, Charlesworth B. 1998. Why sex and recombination? Science 281:1986-1990.

Basson M E, Thorsness M, Rine J. 1986. Saccharomyces cerevisiae contains two functional genes encoding 3-hydroxy-3-methylglutaryl-coenzyme A reductase. Proceedings of the National Academy of Sciences USA 83:5563-5567.

Becskei A, Serrano L. 2000. Engineering stability in gene networks by autoregulation. Nature 405:590593.

Bergman A, Siegal M L. 2003. Evolutionary capacitance as a general feature of complex gene networks. Nature 424:549-552.

Bernard C. 1865. Introduction à l'Étude de la Médecine Expérimentale. Paris: Baillière.[Translated by H C Green. 1957. An Introduction to the Study of Experimental Medicine. New York: Dover Publications.]

Bernoulli D. 1738. Specimen theoriae novae de mensura sortis. Commentarii Academiae Scientiarum Imperialis Petropolitanae 5:175-192. [Translated by L Sommer. 1954. Exposition of a new theory on the measurement of risk. Econometrica 22:23-36.]

Bhalla U S, Iyengar R. 1999. Emergent properties of networks of biological signaling pathways. Science 283:381-387.

Bonner J T. 1988. The Evolution of Complexity by Means of Natural Selection. Princeton (NJ): Princeton University Press.

Bornholdt S, Sneppen K. 2000. Robustness as an evolutionary principle. Proceedings of the Royal Society of London B 267:2281-2286.

Bradshaw A D. 1991. The Croonian Lecture 1991: Genostasis and the limits of evolution. Philosophical Transactions of the Royal Society of London B 333:289305.

Brodie E D, III. 2000. Why evolutionary genetics does not always add up. Pages 3-19 in Epistasis and the Evolutionary Process, edited by J B Wolf, E D Brodie
III, and M J Wade. Oxford and New York: Oxford University Press.

Burch C L, Chao L. 2004. Epistasis and its relationship to canalization in the RNA Virus $\phi 6$. Genetics 167:559-567.

Cannon W B. 1932. The Wisdom of the Body. New York: W. W. Norton.

Charlesworth B. 1993. Directional selection and the evolution of sex and recombination. Genetical Research, Cambridge 61:205-224.

Charlesworth B. 1998. Adaptive evolution: the struggle for dominance. Current Biology 8:R502-R504.

Charlesworth B, Lande R, Slatkin M. 1982. A neo-Darwinian commentary on macroevolution. Evolution 36:474-498.

Cheverud J M, Routman E J. 1995. Epistasis and its contribution to genetic variance components. Genetics 139:1455-1461.

Clarke G M. 1997. The genetic and molecular basis of developmental stability: the Lucilia story. Trends in Ecology and Evolution 12:89-91.

Clarke G M, McKenzie J A. 1987. Developmental stability of insecticide resistant phenotypes in blowfly: a result of canalizing natural selection. Nature 325:345-346.

Davies A G, Game A Y, Chen Z, Williams T J, Goodall S, Yen J L, McKenzie J A, Batterham P. 1996. Scalloped wings is the Lucilia cuprina Notch homologue and a candidate for the modifier of fitness and asymmetry of Diazinon resistance. Genetics 143:1321-1337.

Davies E K, Peters A D, Keightley P D. 1999. High frequency of cryptic deleterious mutations in Caenorhabditis elegans. Science 285:1748-1751.

Debat V, Alibert P, David P, Paradis E, Auffray J-C. 2000. Independence between developmental stability and canalization in the skull of the house mouse. Proceedings of the Royal Society of London B 267:423-430.

Debat V, David P. 2001. Mapping phenotypes: canalization, plasticity and developmental stability. Trends in Ecology and Evolution 16:555-561.

De Visser J A G M, Hermisson J, Wagner G P, Ancel Meyers L, Bagheri-Chaichian H, et al. 2003. Perspective: evolution and detection of genetic robustness. Evolution 57:1959-1972.

Dobzhansky T. 1971. Genetics of the Evolutionary Process. New York: Columbia University Press.

Dover G. 2000. How genomic and developmental dynamics affect evolutionary processes. BioEssays 22(12):1153-1159.

Dworkin I, Palsson A, Birdsall K, Gibson G. 2003. Evidence that Egfr contributes to cryptic genetic variation for photoreceptor determination in natural 
populations of Drosophila melanogaster. Current Biology 13(21): 1888-1893.

Elena S F, Lenski R E. 2001. Epistasis between new mutations and genetic background and a test of genetic canalization. Evolution 55:1746-1752.

Eshel I, Matessi C. 1998. Canalization, genetic assimilation and preadaptation: a quantitative genetic model. Genetics 149:2119-2133.

Falconer D S, Mackay T F C. 1996. Introduction to Quantitative Genetics. Fourth Edition. New York: Prentice Hall.

Falk R. 2001. Can the norm of reaction save the gene concept? Pages 119-140 in Thinking about Evolution: Historical, Philosophical, and PoliticalPerspectives, edited by R S Singh, C B Krimbas, D B Paul, and J Beatty. Cambridge: Cambridge University Press.

Fares M A, Ruiz-González M X, Moya A, Elena S F, Barrio E. 2002. GroEL buffers against deleterious mutations. Nature 417:398.

Fay D S, Han M. 2000. The synthetic multivulval genes of C. elegans: functional redundancy, Ras-antagonism, and cell fate determination. Genesis 26:279284.

Felsenstein J. 1976. The theoretical population genetics of variable selection and migration. Annual Review of Genetics 10:253-280.

Fenster C B, Galloway L F, Chao L. 1997. Epistasis and its consequences for the evolution of natural populations. Trends in Ecology and Evolution 12:282-286.

Ferguson E L, Horvitz H R. 1989. The multivulva phenotype of certain Caenorhabditis elegans mutants results from defects in two functionally redundant pathways. Genetics 123:109-121.

Fisher R A. 1930. The Genetical Theory of Natural Selection. Oxford: Clarendon Press.[Reprint. 1999. Oxford and New York: Oxford University Press.]

Fontana W, Schuster P. 1998. Continuity in evolution: on the nature of transitions. Science 280:1451-1455.

Frank S A. 1999. Population and quantitative genetics of regulatory networks. Journal of Theoretical Biology 197(3):281-294.

Gass G L, Bolker J A. 2003. Modularity. Pages 260-267 in Keywords and Concepts in Evolutionary Developmental Biology, edited by B K Hall and W M Olson. Cambrigde (MA): Harvard University Press.

Gavrilets S, Hastings A. 1994. A quantitative-genetic model for selection on developmental noise. Evolution 48:1478-1486.

Gerhart J, Kirschner M. 1997. Cells, Embryos and Evolution: Toward a Cellular and Developmental Understanding of Phenotypic Variation and Evolutionary Adaptability. Malden (MA): Blackwell Science.

Gibson G. 1996. Epistasis and pleiotropy as natural properties of transcriptional regulation. Theoretical Population Biology 49:58-89.

Gibson G, Dworkin I. 2004. Uncovering cryptic genetic variation. Nature Reviews Genetics 5:681690.
Gibson G, Hogness D S. 1996. Effect of polymorphism in the Drosophila regulatory gene Ultrabithorax on homeotic stability. Science 271:200-203.

Gibson G, van Helden S. 1997. Is function of Drosophila homeotic gene Ultrabithorax canalized? Genetics 147:1155-1168.

Gibson G, Wagner G P. 2000. Canalization in evolutionary genetics: a stabilizing theory? BioEssays 22(4):372-380.

Gibson G, Wemple M, van Helden S. 1999. Potential variance affecting homeotic Ultrabithorax and Antennapedia phenotypes in Drosophila melanogaster. Genetics 151:1081-1091.

Gilbert S F. 2000. Diachronic biology meets evo-devo: C. H. Waddington's approach to evolutionary developmental biology. American Zoologist 40:729737.

Gimelfarb A. 1989. Genotypic variation for a quantitative character maintained under stabilizing selection without mutations: epistasis. Genetics 123:217228.

Goss P J E, Peccoud J. 1998. Quantitative modeling of stochastic systems in molecular biology by using stochastic Petri nets. Proceedings of the National Academy of Sciences USA 95:6750-6755.

Gu Z, Steinmetz L M, Gu X, Scharfe C, Davis R M, Li $\mathrm{W}-\mathrm{H}$. 2003. Role of duplicate genes in genetic robustness against null mutations. Nature 421:6366.

Hadwiger J A, Wittenberg C, Richardson H E, de Barros Lopes M, Reed S I. 1989. A family of cyclin homologs that control the $\mathrm{G}_{1}$ phase in yeast. Proceedings of the National Academy of Sciences USA 86:6255-6259.

Hall B K. 1992. Waddington's legacy in development and evolution. American Zoologist 32:113-122.

Hall B K. 1999. Evolutionary Developmental Biology. Second Edition. Dordrecht (The Netherlands): Kluwer Academic Publishers.

Hall B K, Olson W M, eds. 2003. Keywords and Concepts in Evolutionary Developmental Biology. Cambridge (MA): Harvard University Press.

Hallgrímsson B, Willmore K, Hall B K. 2002. Canalization, developmental stability, and morphological integration in primate limbs. Yearbook of Physical Anthropology 45:131-158.

Hansen T F, Houle D. 2004. Evolvability, stabilizing selection, and the problem of stasis. Pages 107-129 in The Evolutionary Biology of Complex Phenotypes, edited by M Pigliucci and K Preston. Oxford: Oxford University Press.

Hansen T F, Wagner G P. 2001. Modeling genetic architecture: a multilinear theory of gene interaction. Theoretical Population Biology 59:61-86.

Hartl D L, Dykhuizen D E, Dean A M. 1985. Limits of adaptation: the evolution of selective neutrality. Genetics 111:655-674. 
Hartman J L, IV, Garvik B, Hartwell L. 2001. Principles for the buffering of genetic variation. Science 291:1001-1004.

Hartwell L H, Hopfield J J, Leibler S, Murray A W. 1999. From molecular to modular cell biology. Nature 402 (Supplement):C47-C52.

Hedrick P W. 1986. Genetic polymorphism in heterogeneous environments: a decade later. Annual Review of Ecology and Systematics 17:535-566.

Hermisson J, Wagner G P. 2004. The population genetic theory of hidden variation and genetic robustness. Genetics 168:2271-2284.

Hermisson J, Hansen T F, Wagner G P. 2003. Epistasis in polygenic traits and the evolution of genetic architecture under stabilizing selection. American Naturalist 161:708-734.

Ho M W, Bolton E, Saunders P T. 1983a. Bithorax phenocopy and pattern formation. I. Spatiotemporal characteristics of the phenocopy response. Experimental Cell Biology 51(5):282-290.

Ho M W, Saunders P T, Bolton E. 1983b. Bithorax phenocopy and pattern formation. II. A model of prepattern formation. Experimental Cell Biology 51(5):291-299.

Ho M W, Tucker C, Keeley D, Saunders P T. 1983c. Effects of successive generations of ether treatment on penetrance and expression of the bithorax phenocopy in Drosophila melanogaster. Journal of Experimental Zoology 225:357-368.

Hoffmann A A, Woods R. 2001. Trait variability and stress: canalization, developmental stability and the need for a broad approach. Ecology Letters 4:97101.

Houle D. 1991. Genetic covariance of fitness correlates: what genetic correlations are made of and why it matters. Evolution 45:630-648.

Houle D. 1992. Comparing evolvability and variability of quantitative traits. Genetics 130:195-204.

Houle D. 1998. How should we explain variation in the genetic variance of traits? Genetica 102/ 103:241-253.

Houle D. 2001. Characters as the units of evolutionary change. Pages 109-140 in The Character Concept in Evolutionary Biology, edited by G P Wagner. San Diego (CA): Academic Press.

Imasheva A G, Loeschcke V, Zhivotovsky L A, Lazebny O E. 1997. Effects of extreme temperatures on phenotypic variation and developmental stability in Drosophila melanogaster and Drosophila buzzatii. Biological Journal of the Linnean Society 61:117-126.

Jansen R C, Van Ooijen J W, Stam P, Lister C, Dean C. 1995. Genotype by environment interaction in genetic mapping of multiple quantitative trait loci. Theoretical and Applied Genetics 91:33-37.

Kacser H, Burns J A. 1981. The molecular basis of dominance. Genetics 97:639-666.

Kataoka T, Powers S, McGill C, Fasano O, Strathern J,
Broach J, Wigler M. 1984. Genetic analysis of yeast RAS1 and RAS2 genes. Cell 37:437-445.

Kauffman S A. 1993. The Origins of Order: Self-Organization and Selection in Evolution. Oxford and New York: Oxford University Press.

Kawecki T J. 2000. The evolution of genetic canalization under fluctuating selection. Evolution 54:1-12.

Kitcher P. 2001. Battling the undead: how (and how not) to resist genetic determinism. Pages 396-414 in Thinking about Evolution: Historical, Philosophical, and Political Perspectives, edited by R S Singh, C B Krimbas, D B Paul, and J Beatty. Cambridge: Cambridge University Press.

Lande R. 1980. Genetic variation and phenotypic evolution during allopatric speciation. American Naturalist 116:463-479.

Laubichler M D. 2000. Homology in development and the development of the homology concept. American Zoologist 40:777-788.

Layzer D. 1980. Genetic variation and progressive evolution. American Naturalist 115:809-826.

Leamy L J, Routman E J, Cheverud J M. 1998. Quantitative trait loci for fluctuating asymmetry of discrete skeletal characters in mice. Heredity 80:509518.

Lerner I M. 1954. Genetic Homeostasis. Edinburgh: Oliver and Boyd; New York: Wiley.

Lenski R E, Ofria C, Collier T C, Adami C. 1999. Genome complexity, robustness and genetic interactions in digital organisms. Nature 400:661-664.

Levin D A. 1970. Developmental instability and evolution in peripheral isolates. American Naturalist 104:343-353.

Lewontin R C. 1956. Studies on homeostasis and heterozygosity I. General considerations. Abdominal bristle number in second chromosome homozygotes of Drosophila melanogaster. American Naturalist 90:237-255.

Lewontin R C. 1974a. The Genetic Basis of Evolutionary Change. New York: Columbia University Press.

Lewontin R C. 1974b. Annotation: the analysis of variance and the analysis of causes. American Journal of Human Genetics 26:400-411.

Little J W, Shepley D P, Wert D W. 1999. Robustness of a gene regulatory circuit. EMBO Journal 18(15):4299-4307.

Magwene P M. 2001. New tools for studying integration and modularity. Evolution 55:1734-1745.

Maynard Smith J. 1999. Shaping Life: Genes, Embryos and Evolution. New Haven (CT): Yale University Press.

Maynard Smith J, Burian R, Kauffman S, Alberch P, Campbell J, Goodwin B, Lande R, Raup D, Wolpert L. 1985. Developmental constraints and evolution: a perspective from the Mountain Lake Conference on development and evolution. Quarterly Review of Biology 60:265-287.

Maynard Smith J, Sondhi K C. 1960. The genetics of a pattern. Genetics 45:1039-1050. 
McAdams H H, Arkin A. 1997. Stochastic mechanisms in gene expression. Proceedings of the National Academy of Sciences USA 94:814-819.

McAdams H H, Arkin A. 1999. It's a noisy business! Genetic regulation at the nanomolar scale. Trends in Genetics 15:65-69.

Meiklejohn C D, Hartl D L. 2002. A single mode of canalization. Trends in Ecology and Evolution 17:468473.

Mendel G. 1865. Versuche über Pflanzen-Hybriden. Verhandlungen des Naturforschenden Vereines in Brünn Band IV(1865):3-47.

Milton C C, Huynh B, Batterham P, Rutherford S L, Hoffmann A A. 2003. Quantitative trait symmetry independent of Hsp90 buffering: distinct modes of genetic canalization and developmental stability. Proceedings of the National Academy of Sciences USA 100:13396-13401.

Moreno G. 1994. Genetic architecture, genetic behavior, and character evolution. Annual Review of Ecology and Systematics 25:31-44.

Nijhout H F. 2002. The nature of robustness in development. BioEssays 24(6):553-563.

Nijhout H F, Paulsen S M. 1997. Developmental models and polygenic characters. American Naturalist 149:394-405.

Nowak M A, Boerlijst M C, Cooke J, Maynard Smith J. 1997. Evolution of genetic redundancy. Nature 388:167-171.

Orr H A. 2000. Adaptation and the cost of complexity. Evolution 54:13-20.

Ozbudak E M,Thattai M, Kurtser I, Grossman A D, van Oudenaarden A. 2002. Regulation of noise in the expression of a single gene. Nature Genetics 31:6973.

Pál C, Miklós I. 1999. Epigenetic inheritance, genetic assimilation and speciation. Journal of Theoretical Biology 200:19-37.

Palmer A R. 1994. Fluctuating asymmetry analyses: a primer. Pages 335-364 in Developmental Instability: Its Origins and Evolutionary Implications, edited by $\mathrm{T}$ A Markow. Dordrecht (The Netherlands): Kluwer.

Palmer A R, Strobeck C. 1986. Fluctuating asymmetry: measurement, analysis, patterns. Annual Review of Ecology and Systematics 17:391-421.

Palmer A R, Strobeck C. 1992. Fluctuating asymmetry as a measure of developmental stability: implications of non-normal distributions and power of statistical tests. Acta Zoologica Fennica 191:57-72.

Partridge L, Barton N H. 2000. Evolving evolvability. Nature 407:457-458.

Pigliucci M. 1996. How organisms respond to environmental changes: from phenotypes to molecules (and vice versa). Trends in Ecology and Evolution 11:168-173.

Pigliucci M, Murren C T. 2003. Perspective: genetic assimilation and a possible evolutionary paradox: can macroevolution sometimes be so fast as to pass us by? Evolution 57:1455-1464.

Polak M, Starmer W T. 2001. The quantitative genetics of fluctuating asymmetry. Evolution 55:498-511.

Proulx S R, Phillips P C. 2005. The opportunity for canalization and the evolution of genetic networks. American Naturalist 165:147-162.

Queitsch C, Sangster T A, Lindquist S. 2002. Hsp90 as a capacitor of phenotypic variation. Nature 417:618-624.

Real L A, Ellner S. 1992. Life history evolution in stochastic environments: a graphical mean-variance approach. Ecology 73:1227-1236.

Rendel J M. 1967. Canalisation and Gene Control. London: Logos Press; New York: Academic Press.

Rice S H. 1998. The evolution of canalization and the breaking of Von Baer's laws: modeling the evolution of development with epistasis. Evolution 52:647-656.

Rice S H. 2000. The evolution of developmental interactions: epistasis, canalization, and integration. Pages 82-98 in Epistasis and the Evolutionary Process, edited by J B Wolf, E D Brodie III, and M J Wade. Oxford and New York: Oxford University Press.

Rice S H. 2002. A general population genetic theory for the evolution of developmental interactions. Proceedings of the National Academy of Sciences USA 99:15518-15523.

Roff D A. 1997. Evolutionary Quantitative Genetics. New York: Chapman and Hall.

Rollo C D. 1994. Phenotypes: Their Epigenetics, Ecology and Evolution. London and New York: Chapman and Hall.

Rose M R. 1982. Antagonistic pleiotropy, dominance, and genetic variation. Heredity 48:63-78.

Routman E, Cheverud J M. 1994. Individual genes underlying quantitative traits: molecular and analytical methods. Pages 593-606 in Molecular Ecology and Evolution: Approaches and Applications, edited by B Schierwater, B Streit, G P Wagner, and R DeSalle. Basel (Switzerland): Birkhäuser Verlag.

Rutherford S L. 2000. From genotype to phenotype: buffering mechanisms and the storage of genetic information. BioEssays 22(12):1095-1105.

Rutherford S L. 2003. Between genotype and phenotype: protein chaperones and evolvability. Nature Reviews Genetics 4:263-274.

Rutherford S L, Lindquist S. 1998. Hsp90 as a capacitor for morphological evolution. Nature 396:336342.

Sagaret A. 1826. Considerations sur la productions des hybrides des variantes et des variétés en general, et sur celles de Cucurbitacées en particulier. Annales des Sciences Naturelles, Serie 1 8:294-314.

Savageau M A. 1971. Parameter sensitivity as a criterion for evaluating and comparing the performance of biochemical systems. Nature 229:542544. 
Schank J C, Wimsatt W C. 2001. Evolvability: adaptation and modularity. Pages 322-335 in Thinking about Evolution: Historical, Philosophical, and Political Perspectives, edited by R S Singh, C B Krimbas, D B Paul, and J Beatty. Cambridge: Cambridge University Press.

Scharloo W. 1987. Constraints in selection response. Pages 125-149 in Genetic Constraints on Adaptive Evolution, edited by V Loeschcke. Berlin: SpringerVerlag.

Scharloo W. 1988. Selection on morphological patterns. Pages 230-250 in Population Genetics and Evolution, edited by G de Jong. Berlin: Springer-Verlag.

Scharloo W. 1991. Canalization: genetic and developmental aspects. Annual Review of Ecology and Systematics 22:65-93.

Scheiner S M. 1993. Genetics and evolution of phenotypic plasticity. Annual Review of Ecology and Systematics 24:35-68.

Scheiner S M, Lyman R F. 1991. The genetics of phenotypic plasticity. II. Response to selection. Journal of Evolutionary Biology 3:23-50.

Schlichting C D, Pigliucci M. 1998. Phenotypic Evolution: A Reaction Norm Perspective. Sunderland (MA): Sinauer Associates.

Schmalhausen I I. 1938. The Integrating Factors of Evolution. Leningrad: Nature (Priroda).

Schmalhausen I I. 1949. Factors of Evolution: The Theory of Stabilizing Selection. Philadelphia (PA): Blakiston. [Reprint. 1986. Chicago (IL): University of Chicago Press.]

Siegal M L, Bergman A. 2002. Waddington's canalization revisited: developmental stabiligy and evolution. Proceedings of the National Academy of Sciences USA 99:10528-10532.

Slack J M W. 2002. Timeline: Conrad Hal Waddington: the last Renaissance biologist? Nature Reviews Genetics 3:889-895.

Sollars V, Lu X, Xiao L, Wang X, Garfinkel M D, Ruden D M. 2003. Evidence for an epigenetic mechanism by which Hsp90 acts as a capacitor for morphological evolution. Nature Genetics 33:70-74.

Stearns S C. 1982. The role of development in the evolution of life histories. Pages 237-258 in Evolution and Development, edited by J T Bonner. Berlin: Springer-Verlag.

Stearns S C. 1989a. The evolutionary significance of phenotypic plasticity. BioScience 39:436-445.

Stearns S C. 1989b. Comparative and experimental approaches to the evolutionary ecology of development. Geobios (mémoire spéciale) 12:349-355.

Stearns S C. 1993. The evolutionary links between fixed and variable traits. Acta Palaeontologica Polonica 38(3/4):215-232.

Stearns S C. 2000. Daniel Bernoulli (1738): evolution and economics under risk. Journal of Biosciences. 25(3):221-228.
Stearns S C, Kaiser M, Kawecki T J. 1995. The differential genetic and environmental canalization of fitness components in Drosophila melanogaster. Journal of Evolutionary Biology 8:539-557.

Stearns S C, Kawecki T J. 1994. Fitness sensitivity and the canalization of life-history traits. Evolution 48:1438-1450.

Stearns S C, Koella J C. 1986. The evolution of phenotypic plasticity in life-history traits: predictions of reaction norms for age and size at maturity. Evolution 40:893-913.

Stephens D W, Krebs J R. 1986. Foraging Theory. Princeton $(\mathrm{NJ})$ : Princeton University Press.

Stern C. 1958. Selection for subthreshold differences and the origin of pseudoexogenous adaptations. American Naturalist 92:313-316.

Stern D. 2000. Perspective: evolutionary developmental biology and the problem of variation. Evolution 54:1079-1091.

Sternberg P W, Han M. 1998. Genetics of RAS signaling in C. elegans. Trends in Genetics 14:466-472.

Tautz D. 1992. Redundancies, development and the flow of information. BioEssays 14(4):263-266.

Thatcher J W, Shaw J M, Dickinson W J. 1998. Marginal fitness contributions of nonessential genes in yeast. Proceedings of the National Academy of Sciences USA 95:253-257.

Thoday J M. 1955. Balance, heterozygosity and developmental stability. Cold Spring Harbor Symposium of Quantitative Biology 20:318-326.

Thoday J M. 1958. Homeostasis as a selection experiment. Heredity 12:401-415.

True H L, Lindquist S L. 2000. A yeast prion provides a mechanism for genetic variation and phenotypic diversity. Nature 407:477-483.

True H L, Berlin I, Lindquist S L. 2004. Epigenetic regulation of translation reveals hidden genetic variation to produce complex traits. Nature 431:184-187.

Turelli M. 1988. Phenotypic evolution, constant covariances, and the maintenance of additive variance. Evolution 42:1342-1347.

Van Dongen S, Lens L. 2000. The evolutionary potential of developmental instability. Journal of Evolutionary Biology 13:326-335.

Via S. 1994. The evolution of phenotypic plasticity: what do we really know? Pages 35-57 in Ecological Genetics, edited by L A Real. Princeton (NJ): Princeton University Press.

Waddington C H. 1942. Canalization of development and the inheritance of acquired characters. Nature 150:563-565.

Waddington C H. 1953. Genetic assimilation of an acquired character. Evolution 7:118-126.

Waddington C H. 1956. Genetic assimilation of the bithorax phenotype. Evolution 10:1-13.

Waddington C H. 1957. The Strategy of the Genes: A Dis- 
cussion of Some Aspects of Theoretical Biology. London: Allen and Unwin; New York: MacMillan.

Waddington C H. 1960. Experiments on canalizing selection. Genetical Research, Cambridge 1:140-150.

Waddington C H. 1961. Genetic assimilation. Advances in Genetics 10:257-293.

Wade M J, Winther R G, Agrawal A F, Goodnight C J. 2001. Alternative definitions of epistasis: dependence and interaction. Trends in Ecology and Evolution 16:498-504.

Wagner A. 1996. Does evolutionary plasticity evolve? Evolution 50:1008-1023.

Wagner A. 1999. Redundant gene functions and natural selection. Journal of Evolutionary. Biology 12:116.

Wagner A. 2000a. The role of population size, pleiotropy and fitness effects of mutations in the evolution of overlapping gene functions. Genetics 154:1389-1401.

Wagner A. 2000b. Robustness against mutations in genetic networks of yeast. Nature Genetics 24:355361.

Wagner A, Stadler P F. 1999. Viral RNA and evolved mutational robustness. Journal of Experimental Zoology 285:119-127.

Wagner G P. 1996. Homologues, natural kinds and the evolution of modularity. American Zoologist 36:3643.

Wagner G P, Altenberg L. 1996. Perspective: complex adaptations and the evolution of evolvability. Evolution 50:967-976.

Wagner G P, Booth G, Bagheri-Chaichian H. 1997. A population genetic theory of canalization. Evolution 51:329-347.

Wagner G P, Laubichler M D, Bagheri-Chaichian H. 1998. Genetic measurement theory of epistatic effects. Genetica 102/103:569-580.
Whitlock M C, Phillips P C, Moore F B-G, Tonsor S J. 1995. Multiple fitness peaks and epistasis. Annual Review of Ecology and Systematics 26:601-629.

Wijngaarden P J, Koch P B, Brakefield P M. 2002. Artificial selection on the shape of reaction norms for eyespot size in the butterfly Bicyclus anynana: direct and correlated responses. Journal of Evolutionary Biology 15:290-300.

Wilke C O, Wang J L, Ofria C, Lenski R E, Adami C. 2001. Evolution of digital organisms at high mutation rate leads to survival of the flattest. Nature 412:331-333.

Wilkins A S. 1997. Canalization: a molecular genetic perspective. BioEssays 19(3):257-262.

Wolf J B, Brodie E D, III, Wade M J, eds. 2000. Epistasis and the Evolutionary Process. Oxford and New York: Oxford University Press.

Wolf J B, Frankino W A, Agrawal A F, Brodie E D, III, Moore A J. 2001. Developmental interactions and the constituents of quantitative variation. Evolution 55:232-245.

Woltereck R. 1909. Weitere experimentelle Untersuchungen über Artveränderung, speziell über das Wesen quantitativer Artunterschiede bei Daphniden. Verhandlungen der Deutschen Zoologischen Gesellschaft 1909:110-172.

Wright S. 1934. Physiological and evolutionary theories of dominance. American Naturalist 68:24-53.

Wright S. 1977. Evolution and the Genetics of Populations, Volume 3: Experimental Results and Evolutionary Deductions. Chicago (IL): University of Chicago Press.

Wu R. 1998. The detection of plasticity genes in heterogeneous environments. Evolution 52:967-977.

Zakharov V M. 1992. Population phenogenetics: analysis of developmental stability in natural populations. Acta Zoologica Fennica 191:7-30. 